\title{
Modeling panel detection frequencies by queuing system theory: An application in gas chromatography olfactometry
}

\author{
JOHANNES H. F. BULT and BRAM VAN PUTTEN \\ Wageningen University and Research Center, Wageningen, The Netherlands \\ HENDRIK N. J. SCHIFFERSTEIN \\ Delft University of Technology, Delft, The Netherlands \\ JACQUES P. ROOZEN and ALPHONS G. J. VORAGEN \\ Wageningen University and Research Center, Wageningen, The Netherlands \\ and \\ JAN H. A. KROEZE \\ Utrecht University, Utrecht, The Netherlands \\ and Wageningen University and Research Center, Wageningen, The Netherlands
}

\begin{abstract}
In continuous vigilance tasks, the number of coincident panel responses to stimuli provides an index of stimulus detectability. To determine whether this number is due to chance, panel noise levels have been approximated by the maximum coincidence level obtained in stimulus-free conditions. This study proposes an alternative method by which to assess noise levels, derived from queuing system theory (QST). Instead of critical coincidence levels, QST modeling estimates the duration of coinciding responses in the absence of stimuli. The proposed method has the advantage over previous approaches that it yields more reliable noise estimates and allows for statistical testing. The method was applied in an olfactory detection experiment using 16 panelists in stimulus-present and stimulus-free conditions. We propose that QST may be used as an alternative to signal detection theory for analyzing data from continuous vigilance tasks.
\end{abstract}

A central question in the domain of the psychology of perception is how to distinguish stimulus-induced responses from responses to noise. In the present article, we use a quantitative approach to estimate noise levels for a panel of subjects responding to a sequence of events in a vigilance task. Traditionally, the theoretical framework that has been used in experiments in which signals have to be distinguished from noise has been signal detection theory (SDT). Since this approach is difficult to apply in vigilance tasks in which stimulus incidences are low, we propose a different framework based on queuing system theory (QST), a framework originally developed to model the dynamics of electronic networks. First, we will introduce the vigilance task paradigm and discuss the disadvantages of using SDT in this context. Then we

This research was funded by the Netherlands Organization for Scientific Research (NWO), by Grant NWO-SGW-575-24.002 awarded to J.H.A.K. The authors thank V. F. Nicola at the University of Twente for inspiring us in the initial phase of this project. Correspondence concerning this article should be addressed to J. H. F. Bult, Wageningen Center for Food Sciences, P.O. Box 557, 6700AN Wageningen, The Netherlands (e-mail: harold.bult@wur.nl).

-This article was accepted by the previous editorial team, headed by Neil Macmillan. will introduce QST and demonstrate how it can be used to model the response behavior of a panel of observers under the assumption that no stimuli are presented. Subsequently, we will demonstrate the use of the framework in an olfactory vigilance experiment in which odorants have to be detected at a sniffing port. Panelists' responses that are generated in the absence of stimuli are used to estimate the parameters of the QST model in two distinct stimulus contexts: stimulus-free (SF) sessions and SF windows of stimulus-present (SP) sessions. Finally, in a simulation study, we derive a heuristic that yields variances of panel response lengths at each level of coinciding responses. With these variances, critical values of response lengths are calculated to enable statistical testing of observed response coincidences.

\section{Vigilance Tasks and SDT}

The vast majority of human psychological experiments involving stimulus presentations have been conducted using highly structured, time-controlled presentation trials. Vigilance tasks form an exception to that rule. In its elementary form, a vigilance task consists of a sequence of randomly timed presentations of identical stimuli. The subject is asked to give a simple response, generally by pressing a button, whenever he or she believes that the 
critical event - a stimulus presentation-has occurred. Two presentation modes can be distinguished: Either stimuli may be presented during distinct observation intervals, or stimuli may be presented at any point in time, without observation intervals being defined. The former, which is the most common mode, can be described as a discrete-events task, whereas the latter is known as a continuous or free-response task (Egan, Greenberg, \& Schulman, 1961a, 1961b). Although the vigilance paradigm covers a small portion of the literature on perception, it represents a wide range of daily life situations, ranging from motorists checking their cars' instrument panels to lifeguards watching over bathers' safety. Vigilance tasks require sustained attention from the observer: The critical event requiring a response might occur at any moment. The incidence of critical events, however, is often very low. It is not by the hour that a bather's life is in danger, nor does a car's oil pressure drop drastically once every day. Task difficulty is high when critical event intensities are weak or when events have to be discriminated from background noise. A bather's call for help might easily go unnoticed in the cacophony of sounds at the beach.

SDT offers a framework for the quantification of the ability to detect weak stimuli or to discriminate stimuli from noise (Green \& Swets, 1966; Swets, 1961). Since the early 1960 s, SDT has been applied in vigilance experiments, in both the auditory (Egan et al., 1961a) and the visual (Mackworth \& Taylor, 1963) domain. Its usefulness is still recognized to date (Craig, 1987; See, Warm, Dember, \& Howe, 1997). SDT assumes that each observation is projected on an arbitrary sensory continuum. The location on the continuum is determined by the projected stimulus (signal) intensity and normally distributed noise. Assuming that noise $(\mathrm{N})$ and stimulus + noise ( $\mathrm{SN}$ ) magnitudes have equal variances, the detectability index $d^{\prime}$ of a stimulus is defined as the difference between the means of the probability density distributions of $\mathrm{N}$ and $\mathrm{SN}$, expressed in standard deviation units. Under the assumption that $\mathrm{N}$ and $\mathrm{SN}$ magnitudes are Gaussian distributed for a given stimulus condition, the detectability $d^{\prime}$ can be calculated from observed proportions of true detections (hits in SDT) and false detections (false alarms in SDT) (Green \& Swets, 1966). The probability that an observer detects a stimulus correctly increases with increasing $d^{\prime}$. In addition, this probability depends on an arbitrary, observer-dependent critical value on the sensory continuum $\left(x_{\mathrm{c}}\right)$, which is the lower bound sensation magnitude for the observer to decide that a stimulus has been presented. A higher $x_{\mathrm{c}}$ will result in a lower probability of yes responses (both hits and false alarms) and a higher probability of no responses (both true rejections and misses). This observer-dependent decision criterion is expressed by the likelihood ratio $\beta=P\left(x_{\mathrm{c}} \mid \mathrm{SN}\right) /$ $P\left(x_{\mathrm{c}} \mid \mathrm{N}\right)$, which equals the probability of a hit divided by the probability of a false alarm. Since the average of the SN distribution is at least equal but usually higher than the average of the $\mathrm{N}$ distribution, high decision criteria
( $\beta>1$ ) generally reflect high $x_{\mathrm{c}}$ (exceeding the average of the SN and the $\mathrm{N}$ distributions).

However useful SDT may be in a wide range of perception experiments, free-response vigilance tasks are a special case in which the use of SDT has some serious disadvantages. During a vigil, a stimulus may occur occasionally and at any point in time. The low presentation rate, in combination with the lack of temporal structure, introduces difficulties with respect to the qualification of responses in terms of SDT. On the one hand, SDT requires sufficiently high rates of both hits and false alarms to ensure a reliable estimation of $d^{\prime}$. On the other hand, the lack of structured presentation intervals produces difficulty in deciding whether a delayed affirmative response event is still to be regarded as a hit or a false alarm (Watson \& Nichols, 1976). Furthermore, in classical signal detection experiments, signals generally are presented at a constant duration and last less than $1,000 \mathrm{msec}$, which is not comparable to many practical situations in which stimulus duration is highly variable, influencing the probability of stimulus detection.

In the present study, we will present an alternative, QST-based conceptual approach to estimating noise levels in continuous vigilance tasks, in which combined panel responses given in the absence of stimuli are used. Only false alarms serve as input for the model. Hence, no hits are needed, and no structured presentation intervals need to be defined. After a noise level is determined, observed panel responses from SP tasks can be tested against the null hypothesis that no stimuli were present. In QSTmodeling, response length relates directly to detection probability, which respects the variability of stimulus duration under natural conditions and thus improves its ecological validity in comparison with SDT.

\section{QST Applied to Vigilance Tasks}

Queuing systems are described as systems of flowthat is, systems "in which some commodity flows, moves, or is transferred through one or more finite-capacity channels in order to go from one point to another" (Kleinrock, 1975, p. 3). Examples are the flow of automobile traffic through a road network, the transmission of telephone messages through a telecommunication network, or the flow of data through a time-sharing computer system. In terms of vigilance tasks, the flow system is reflected by panelists' processing of responses to a specific sequence of stimuli. The number of members in the panel defines the channel capacity, and the commodity is formed by the panelists' responses to the stimuli presented. The state of the flow system is the number of panelists responding simultaneously, which is expressed as the number of coinciding responses: the coincidence level. Transitions between coincidence levels follow individual response onsets and offsets. An example of how the individual responses of four panelists are combined to form coincidence levels is given in Figure 1.

Under the assumption that all responses are false alarms (i.e., no stimuli are presented), QST can formalize re- 
sponse behavior by modeling the probabilities of coincidence levels. This allows for the definition of panel noise levels of coinciding responses. From false alarm responses, two measures are used: the length of responses and the latency between consecutive responses (see Figure 1). Response length and response latency together determine response incidence. If the average latency increases, response incidence decreases. On the other hand, an increase of response length reduces response incidence. However, since response length is generally short, as compared with response latency, changes in response length will have a smaller impact on response incidence than will changes in response latency.

To model coincidence level probability in QST terms, two requirements should be met with respect to the model. The QST model should allow for coincidence levels that range from zero up to and including the panel size. In addition, each response action should instantly produce a transition in coincidence level. This implies a channel capacity that equals the number of panelists, so that waiting times are zero. QST modeling makes two assumptions with respect to the occurrence of responses in SF sessions. First, a transition in coincidence level is as- sumed to take place one step at a time. Consequently, the model assumes that two panelists never initiate or end a response at exactly the same moment. Second, transition probability as a function of time is defined by the current coincidence level only. It does not depend on the past coincidence levels or on the length of the time interval at the actual coincidence level. In other words, the queuing system is memoryless.

A simple response system that fits these requirements is the response onset-only system. An imaginary person presses a button repeatedly although no stimuli are presented. Every buttonpress signifies a response onset. Following the memoryless property of the system, the probability density function of the length of the time period between two consecutive onsets can be described by the exponential decay function (Kleinrock, 1975):

$$
a(t)=\lambda e^{-\lambda t}, t \geq 0,
$$

where $t$ is the time since the start of the observation, $a(t)$ is the probability density at time $t$, and the onset rate $\lambda$ is the expected number of button hits per unit of time. The exponential decay function satisfies the assumption that the probability of a response onset as a function of

\section{Individual responses}
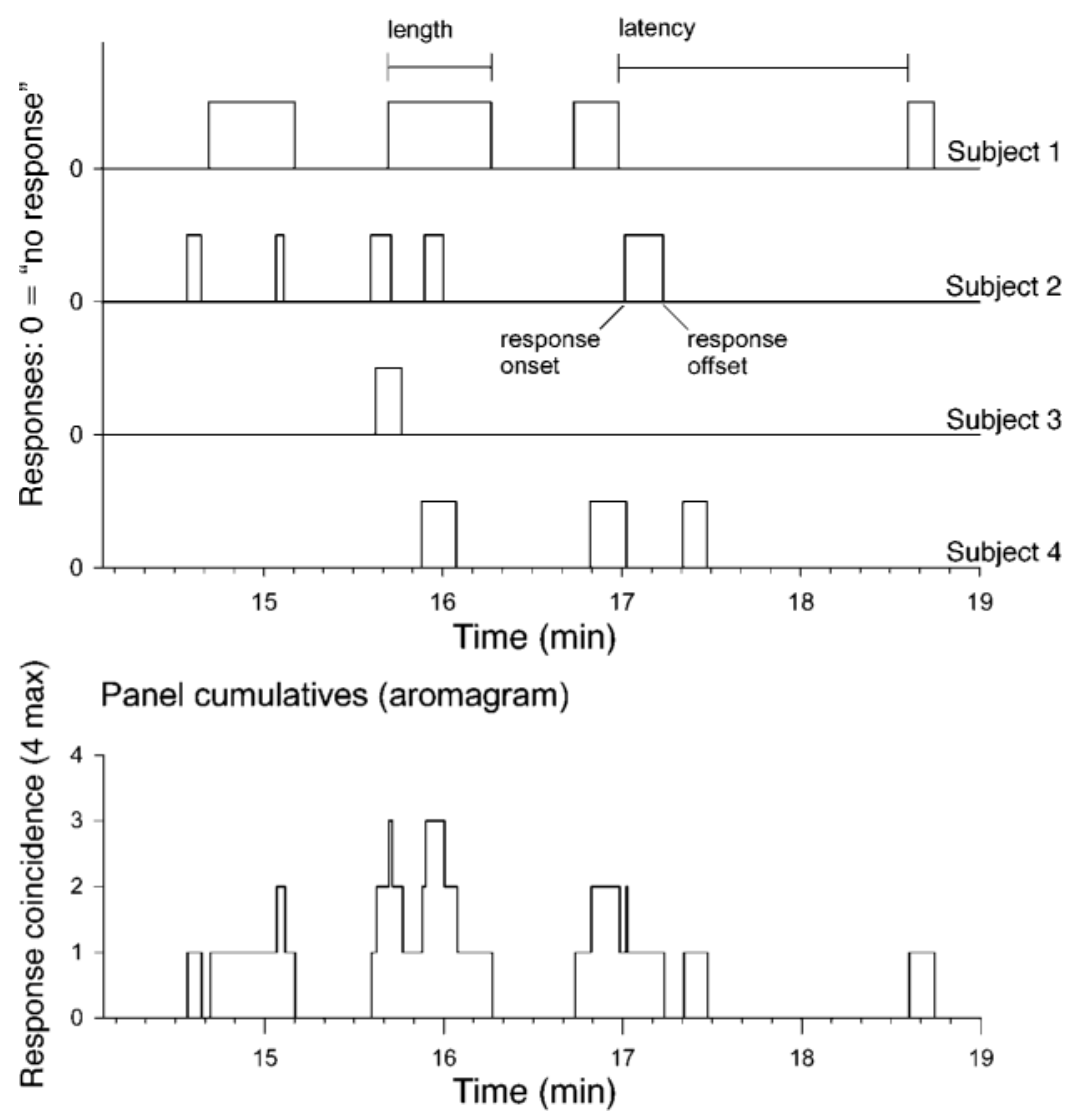

Figure 1. Determining coincidence levels from individual responses. The upper panel shows responses generated by 4 subjects to identical stimulus sequences. The lower panel shows the aggregate coincidence levels as cumulatives on the time axis. 
time $t$ depends neither on the moment that registration started nor on the number of previous responses. If the response onset times are exponentially distributed, the probability of the number of responses being $k$ at time $t$ is Poisson distributed:

$$
P_{k}(t)=\frac{(\lambda t)^{k}}{k !} e^{-\lambda t}, \quad(k \geq 0, t \geq 0) .
$$

To account for response offsets, a measure of response length is introduced, reflecting the interval during which a subject perceives an ongoing stimulus. In SF conditions requiring little stimulus processing, the exponential function (Equation 1) accurately describes response length distributions (Luce, 1986). The probability density function of the length of a single response can be defined using the offset rate $\mu$, which equals the expected mean number of response intervals per unit time. The expected mean length of a single response then equals the reciprocal of $\mu$ :

$$
E(\overline{\text { length }})=\frac{1}{\mu} \text {. }
$$

The rate at which a panel initiates responses depends on the panel size, the individual onset rates, and the number of panelists already responding. For instance, the probability that any panelist initiates a response, given that all panelists are currently responding, is zero. This rate will increase with the number of nonresponding panelists available. Given a panel of size $M$ and assuming that individual onset rates are identical and additive, the collective onset rate of a panel in which $k$ panelists are currently responding $\left(\lambda_{k}\right)$ can be calculated from the individual rates for any given coincidence level $k$ :

$$
\lambda_{k}=(M-k) \lambda, \text { for } 0 \leq k \leq M .
$$

Under the same assumptions, the collective offset rate $\mu_{k}$ can be calculated by

$$
\mu_{k}=k \mu, \text { for } 0 \leq k \leq M .
$$

From Equations 1, 4, and 5, Kleinrock (1975) arrives at a model describing the probability that $k$ out of $M$ subjects are responding simultaneously at an arbitrary moment in time, given that no stimuli are presented:

$$
P_{k}=\frac{\left(\frac{\lambda}{\mu}\right)^{k}\left(\frac{M}{k}\right)}{\left(1+\frac{\lambda}{\mu}\right)^{M}}\left(0 \leq k \leq M \text { and } \sum_{k=0}^{M} P_{k}=1\right) .
$$

Given $\lambda$ and $\mu$, the model can be used to calculate the expected proportion of the total session length at which $k$ subjects will be responding simultaneously, under the condition that no stimuli are present. The expected cumulative length of time $\left(T_{k}\right)$ at coincidence level $k$ can then be calculated by multiplying $P_{k}$ by the length of the experimental session $\left(l_{\text {session }}\right)$ :

$$
T_{k}=P_{k} \cdot l_{\text {session }} \text {. }
$$

Following

$$
\sum_{k=0}^{M} P_{k}=1, T_{k}
$$

are also linearly dependent because

$$
\sum_{k=0}^{M} T_{k}=l_{\text {session }} .
$$

Parameters $\lambda$ and $\mu$ can be assessed empirically. For each subject, the offset rate $\mu$ is estimated by the reciprocal of the average length of responses and can be calculated by

$$
\hat{\mu}=(\overline{\text { length }})^{-1}=\frac{n_{\text {responses }}}{\sum \text { length }},
$$

where length is the response length (see Figure 1) and $n_{\text {responses }}$ the number of responses involved. Individual onset rate is estimated by the reciprocal of the average latency and can be calculated by

$$
\hat{\lambda}=(\overline{\text { latency }})^{-1}=\frac{n_{\text {responses }}}{l_{\text {session }}-\sum \text { length }},
$$

where latency is the length of the interval between successive responses (see Figure 1). In the present study, we will use the results of each individual panelist to estimate individual onset and offset rates. In addition, we will estimate overall panel onset and offset rates. The latter requires concatenating responses from several individuals as if these were generated by one person during a session of length $M \cdot l_{\text {session }}$.

By substituting the empirical estimates $\hat{\lambda}$ and $\hat{\mu}$ (Equations 8 and 9) in Equation 6, estimates of $P_{k}\left(\hat{P}_{k}\right)$ can be obtained. However, it is virtually impossible to derive an exact probability distribution of $\hat{P}_{k}$ analytically from the exponential probability distributions of $\hat{\lambda}$ and $\hat{\mu}$. Instead, we simulated SF sessions under varying conditions of session length, $\lambda$, and $\mu$. The resulting distributions of $\hat{T}_{k}$ were used to model $\operatorname{Var}\left(\hat{T}_{k}\right)$ as a function of session length, $\lambda$, and $\mu$. Results of these simulations will be discussed in the simulation section of this article. First, we will present a study of noise estimates in an olfactory vigilance task - that is, gas chromatography olfactometry (GCO).

\section{Gas Chromatography Olfactometry}

Flavor chemists face the challenge of discriminating odorous components from the many odorless components in mixtures of volatiles constituting food aromas. Generally, gas chromatography is used to physically separate mixture constituents. It entails the pressurized transfer of mixture constituents through a capillary column under controlled temperature conditions. The time that each component needs to pass through the column depends on its specific physical-chemical interaction with the column lining. On release from the column, each component is quantified by a detector and identified by mass spectrometry. The mass release function 
typically shows a steep, approximately linear incline to a maximum, followed by a more gradual and tailing decay to zero. We define the stimulus onset time as the point in time at which the mass function starts to incline and the offset time as the point in time at which the mass function returns to zero. Typically, between 5 and $45 \mathrm{sec}$ elapse between stimulus onset and offset.

However, the capacity of volatiles to invoke odor sensations at a given concentration level varies strongly, due to large differences in detection thresholds between odorants. Hence, relative quantities of the components in the mixture are poor indicators of their relative contributions to the mixture's aroma. A better estimate of each component's contribution to the aroma may be obtained by sensory evaluation of the separated constituents. Therefore, a method combining gas chromatography with sensory evaluation was developed, enabling the assessment of the olfactory impact of mixture constituents. With this method, called GCO, subjects sniff the effluents of the gas chromatograph (GC) in an effort to detect and characterize the odor-active chemicals (Dravnieks \& O'Donnell, 1971).

To quantify the sensory impact of the effluents, several sensory methods have been proposed. Grosch (2001) distinguishes three categories of GCO techniques. (1) Dilution methods use the number of times a sample needs to be diluted until it can no longer be detected as a measure of odor impact. Examples of this approach are charm analysis (Acree, Barnard, \& Cunningham, 1984) and aroma extract dilution analysis (Ullrich \& Grosch, 1987). (2) Detection frequency (DF) methods employ the number of coinciding panel detection responses to a stimulus as an indicator of its odor impact (Bult, Schifferstein, Roozen, Voragen, \& Kroeze, 2001; Ott, Fay, \& Chaintreau, 1997; Pollien et al., 1997; van Ruth \& Roozen, 1994). This method is also referred to as olfactory global analysis (Grosch, 2001; Le Guen, Prost, \& Demaimay, 2000). It reflects the vigilance task described above. Finally, (3) intensity rating methods such as the Osme method (Da Silva, Lundahl, \& McDaniel, 1994; McDaniel, Miranda-Lopez, Watson, Micheals, \& Libbey, 1990) use panelists' intensity ratings of undiluted GC effluents to assess their odor impact. The three methods generate highly comparable results when used to determine the main contributors to an aroma (Le Guen et al., 2000). However, none of these methods allows statistical testing of positive stimulus detections. In the present study, we attempt to improve the DF method by proposing a method that tests whether the observed DFs fit a distribution of DFs that is expected to occur in the absence of stimuli.

To the present, DF methods have been employed to obtain measures of odor impact without assessing the reliability of positive identifications. Users of the DF method have used arbitrary noise levels (Le Guen et al., 2000), or they have conducted SF sessions to determine the level at which coincidences in SP sessions should be interpreted as noise (van Ruth \& Roozen, 1994; van
Ruth, Roozen, \& Cozijnsen, 1995, 1996). In the latter approach, the highest response coincidence level encountered in SF sessions was considered the critical noise level for the SP sessions. However, this approach suffers from fundamental shortcomings. First of all, the critical noise level estimates are affected by session length. If the probability that 12 responses coincide at least once during a 1-h session with a panel of 15 subjects equals $10^{-5}$, the aggregate probability of finding 12 coinciding responses at least once during 100 consecutive hours equals $99 \cdot 10^{-5}$, assuming that the subsequent responses of panelists are independent. Second, the use of SF sessions to estimate noise levels presupposes that the tendency to generate false alarms is independent of stimulus frequency. However, this assumption is contradicted by experimental findings from vigilance studies (Swets, 1977; Vickers, Leary, \& Barnes, 1977; Warm, Dember, \& Parasuraman, 1991). Subjects appear to adjust their decision criterion depending on the perceived stimulus probability: Response frequencies decrease when perceived stimulus probabilities decrease, and response frequencies increase when perceived stimulus probabilities increase (Colquhoun \& Baddeley, 1967; Williges, 1969).

In the following empirical section, we will apply QST modeling to the results from a continuous vigilance task, in which a panel had to detect odorants at a sniffing port connected to a GC outlet. We use the classical DF approach to assess noise levels and compare the results with best estimates of $\hat{T}_{k}$ calculated by QST modeling. Response coincidence probabilities are estimated for SF sessions and for SF windows in SP sessions, using model parameters $\lambda$ and $\mu$, which are estimated for the two conditions separately.

\section{OLFACTORY EXPERIMENT}

\section{Method}

Subjects. Sixteen paid volunteers from the local community, 4 males and 12 females, served as subjects. Their ages ranged from 20 to 53 years (average, 29 years). Twelve were experienced subjects who had participated in olfactory attribute-rating experiments, discrimination tasks, and GCO experiments over the course of 2 years. Four new subjects were selected and familiarized with the GCO method during a 45-min training session. The selection criteria for all the subjects comprised the ability to generate and use refined odor attributes and intersubject coherency in the use of graphic rating scales. The subjects were naive as to the objectives of the experiment. All were nonsmokers and had no history of olfactory dysfunction. The subjects were in good health and gave written informed consent.

Stimuli. A model mixture was used, consisting of nine chemical components that are commonly found in natural apple aromas. The concentrations of seven components (Table 1, numbers 2, 3, 4, 5, 6, 8 , and 9) matched the relative concentrations of these components in the saturated headspace of a bottle containing fresh apple juice (Bult et al., 2001). The other two odorants, diacetyl and ethyl 2methylbutanoate are also found in apples (Maarse, Visscher, Willemsens, \& Boelens, 1989). They were added because of their distinct smells and because they would increase the span of stimulus onset times. Their concentrations would produce intensities in the same range as the other components (see Table 1). The components were 
Table 1

Stimulus Onset Times, Reported Threshold Concentrations in Water and Air of the Odorants Used, and Their Mean Sniffing Port Masses in the Present Study

\begin{tabular}{|c|c|c|c|c|c|c|c|}
\hline \multirow[b]{2}{*}{$\begin{array}{l}\text { Peak } \\
\text { Index }\end{array}$} & \multirow[b]{2}{*}{$\begin{array}{l}\text { Component } \\
\text { Name }\end{array}$} & \multirow{2}{*}{$\begin{array}{l}\text { Onset } \\
\text { Time }^{\mathrm{a}} \\
(\mathrm{min})\end{array}$} & \multicolumn{2}{|c|}{$\begin{array}{l}\text { Reported Detection } \\
\text { Threshold Concentration }\end{array}$} & \multirow{2}{*}{$\begin{array}{l}\text { In } 10-\mathrm{ml} \\
\text { Stock } \\
\text { Solution } \\
\text { (mg) }\end{array}$} & \multirow{2}{*}{$\begin{array}{c}\text { Mass at } \\
\text { Sniffing } \\
\text { Outlet }^{\mathrm{k}} \\
\text { (ng) }\end{array}$} & \multirow{2}{*}{$\begin{array}{c}\text { Ratio Mass at } \\
\text { Sniffing Outlet to } \\
\text { Threshold in Air } \\
(\mathrm{L})^{1}\end{array}$} \\
\hline & & & $\begin{array}{c}\text { Water } \\
\text { (ppb vol/vol) }\end{array}$ & $\begin{array}{c}\text { Air } \\
(\mathrm{ng} / \mathrm{L})\end{array}$ & & & \\
\hline 1 & diacetyl & 7.21 & $1.4^{\mathrm{b}}$ & $5.0^{\mathrm{b}}$ & 0.25 & 0.74 & 0.15 \\
\hline 2 & propyl acetate & 11.25 & - & $200-7000^{\mathrm{c}}$ & 4.44 & 13.32 & $0.0019-0.067$ \\
\hline 3 & isobutyl acetate & 14.17 & $10^{\mathrm{d}}$ & $1.7-17^{\mathrm{c}}$ & 8.68 & 26.04 & $1.5-15$ \\
\hline 4 & hexanal & 15.20 & $4.5^{\mathrm{e}}$ & $30-53^{f}$ & 25.02 & 75.06 & $1.4-2.5$ \\
\hline 5 & butyl acetate & 16.40 & $66 \mathrm{~g}$ & $30-180^{c}$ & 39.69 & 119.07 & $0.66-3.7$ \\
\hline 6 & trans-2-hexenal & 18.30 & $17^{\mathrm{e}, \mathrm{g}}$ & $340^{\mathrm{c}}$ & 21.15 & 63.45 & 0.19 \\
\hline 7 & ethyl 2-methyl butanoate & 19.01 & $0.006-0.008^{\mathrm{h}, \mathrm{i}}$ & $0.1-0.3^{\mathrm{j}}$ & 0.22 & 0.65 & $2.2-6.5$ \\
\hline 8 & 2-methyl-1-butyl acetate & 20.48 & $5 \mathrm{~g}$ & $90-200^{c}$ & 35.04 & 105.12 & $0.52-1.2$ \\
\hline 9 & hexyl acetate & 25.68 & $2 \mathrm{~g}$ & $2.3^{\mathrm{c}}$ & 43.50 & 130.50 & 56 \\
\hline
\end{tabular}

aNormalized to stimulus onset times of a selected reference session (see text). ${ }^{b}$ Hall \& Andersson, $1983 . \quad{ }^{c}$ van Gemert \& Nettenbreijer, 1977 ; thresholds were calculated mostly from water concentrations in water-headspace systems, employing either empirical or literature partition coefficients. ${ }^{\mathrm{d} O n g} \&$ Acree, 1998. $\quad$ eButtery \& Ling, 1998. $\quad{ }^{\mathrm{f}}$ Rychlik, Schieberle, \& Grosch, 1998. gFlath, Black, Guadagni, McFadden, \& Schultz, 1967. hTakeoka et al., 1998. iTakeoka, Buttery, Turnbaugh, \& Teranishi, 1991. jKollmannsberger \& Berger, $1992 . \quad$ kEquals $40 \%$ of the total amount that was injected on column. ${ }^{1}$ Actual concentrations of inhaled components depend on the sniff vigor, and therefore, ratios are merely relative measures of odor impact.

dissolved in $10.0 \mathrm{ml}$ of $n$-pentane $\left(4^{\circ} \mathrm{C}\right)$ at the concentrations shown in Table 1 and were stored at $4^{\circ} \mathrm{C}$ after preparation. Immediately before the start of a sensory experiment, $0.075 \mu \mathrm{L}$ of this stock solution was transferred to a glass tube containing Tenax (Tenax TA, 35/60 mesh; Alltech Nederland, Zwijndrecht, the Netherlands), a granulated absorbent material.

Stimulus production. The injected components were thermally desorbed from Tenax at $260^{\circ} \mathrm{C}$ for $300 \mathrm{sec}$ and were cryofocused at $-120^{\circ} \mathrm{C}$ by a cold trap/thermal desorption device (Carlo Erba TDAS 5000; Interscience, Breda, the Netherlands). Components were separated on a DB1 column (J\&W Scientific, $60 \mathrm{~m} \times 0.25 \mathrm{~mm}$ i.d.; film thickness $=0.25 \mu \mathrm{m})$. Oven temperature was initially kept at $40^{\circ} \mathrm{C}(4 \mathrm{~min})$ and then was increased to $75^{\circ} \mathrm{C}\left(3.0^{\circ} \mathrm{C} / \mathrm{min}\right)$ and, subsequently, to $80^{\circ} \mathrm{C}\left(1.0^{\circ} \mathrm{C} / \mathrm{min}\right)$. After a final increase to $272^{\circ} \mathrm{C}$ $\left(15^{\circ} \mathrm{C} / \mathrm{min}\right)$, oven temperature was kept at this temperature for another $5 \mathrm{~min}$. This program allowed for an optimal separation of stimulus onset times. Column effluents were split. The ducts from splitter to sniffing outlets were kept at oven temperature to prevent condensation of volatiles. Of the total flow, $20 \%$ was directed to a flame ionization detector (FID), while the two sniffing outlets each received $40 \%$. Column effluents were mixed with humidified nitrogen at the sniffing outlet. On presentation, odorants were expected to have variable intensities, as may be inferred from the ratio of odorant masses at the sniffing outlet to their corresponding odor thresholds (Table 1).

Procedure. The experiment was part of a larger GCO study that was originally undertaken to investigate the effects of stimulus onset time and stimulus order on sensory evaluations. Originally, two ordering conditions were used, each comprising a different stimulus order and different stimulus onset times. Per subject, four sessions were randomly assigned to one ordering category, and four other sessions to the other ordering category. In the present study, data were used from one SP session only, containing the same stimulus order for each subject. For each subject, one SF session was included to provide a reference for response behavior due to noise. This SF session could occur on any experimental day, with the exception of the first day, at which only SP sessions were run. On average, the SF session occurred on Day 4.0. One SP session was chosen at random from the four sessions with the same stimulus orders. In the group of 16 subjects, this session occurred 6 times before an SF session and 10 times after an SF session and occurred, on average, on Day 4.2 in the sequence.
During a session, 1 or 2 subjects were seated with the nose positioned at the sniffing outlet connected to the GC. A blind prevented visual contact between subjects, who were not allowed to interact with each other in any way. To ensure that the solvent peak would not be inhaled, sniffing started 6 min after the initiation of the GC procedure. Sniffing analyses finished after $36 \mathrm{~min}$. Hence, all the subjects completed sniffing sessions of $30 \mathrm{~min}$. The subjects were instructed to inhale slowly through the nose at an even pace and to exhale at a higher pace. In this way, the dilution of sniffed odorants with the surrounding air was minimized, while the net observation time was maximized. To respond as quickly as possible to stimulus events, the subjects chose one easily accessible key on the first day, usually the space bar on the keyboard placed in front of them. Throughout the experiment, the subjects were instructed to strike this key at stimulus onset and again at stimulus offset. This procedure was chosen for the following reasons. First, it compels subjects to attach equal significance to the decision that a stimulus has started and to the decision that a stimulus has terminated. Second, this procedure is physically less fatiguing than when one key has to be pressed continuously until the stimulus stops. Finally, this method is less prone to registration errors, because it is easier to strike a key briefly than to keep it pressed continuously. In between the two keystrokes, visual feedback on the computer screen indicated that a response was being recorded. No cues as to the occurrence of a stimulus were given. After each second keystroke, the subjects had to rate odor quality by selecting descriptors from a list. These ratings are not analyzed in the present study. The registration of responses was electronically synchronized with FID registrations. Room temperature was kept at $21^{\circ} \mathrm{C}$. The air inside the room was ventilated and filtered.

Data analysis. Model parameters $\lambda$ and $\mu$ were estimated according to Equations 8 and 9 for both SF and SP sessions. For SF sessions, these estimates could be calculated using all response events that were generated during the session. The SP data, however, were first trimmed by omitting all response intervals that coincided with stimulus presentations, as indicated by FID readings. Because subjects may persist responding after the stimulus itself has disappeared, we prolonged SP intervals by $20 \mathrm{sec}$. Parameters of the QST model were then estimated on the basis of the responses given during the SF windows of the SP sessions. To illustrate the impact that stimuli have on parameter estimates, QST parameters were also calculated for SP sessions without trimming SP windows. 
Although the sequential order of component peaks did not differ between sessions, the stimulus onset times of identical FID peaks varied slightly. This was due to small variations between sessions in oven temperature and column gas pressure. In the present study, the average absolute deviation from the mean stimulus onset time was $8.5 \mathrm{sec}$. Because subjects' response times for identical components will vary accordingly between sessions, responses may fail to coincide in time. The example in Figure 2 shows stimulus onset times in the upper reference session that deviate for Stimulus 9 in Session A and for Stimuli 6 and 7 in Session B. The responses to these stimuli therefore fail to coincide. To correct for effects of stimulus onset time variation on resulting response coincidences, we normalized the stimulus onset times and response times with respect to the session that showed the least cumulated deviation from mean stimulus onset times (the reference session). First, stimulus onset times of identical stimuli in different sessions were assigned the onset time for that stimulus in the reference session - that is, $t_{\mathrm{a}, 1}=t_{\mathrm{r}, 1}$ and $t_{\mathrm{b}, 1}=t_{\mathrm{r}, 1}$ (Figure 2). Onset and offset response events that took place in between two subsequent stimulus onsets were then corrected by linear interpolation following the corresponding stimulus onset times in the reference session. Formally, response event times $\left(t_{\text {event }}\right)$ in between the onset times of stimuli $n-1$ and $n$ in Session A $\left(t_{\mathrm{a}, \mathrm{n}-1}\right.$ and $\left.t_{\mathrm{a}, \mathrm{n}}\right)$ are assigned the normalized onset times:

$$
\operatorname{norm}\left(t_{\text {event }}\right)=t_{r, n-1}+\frac{\left(t_{\text {event }}-t_{a, n-1}\right)}{\left(-t_{a, n}-t_{a, n-1}\right)} \cdot\left(t_{r, n}-t_{r, n-1}\right),
$$

with $t_{r, n-1}$ and $t_{r, n}$ being the stimulus onset times in the reference session corresponding to $t_{a, n-1}$ and $t_{a, n}$, respectively, in Session A. For events that took place before the first stimulus onset, $t_{a, n-1}$ and $t_{r, n-1}$ were assumed to equal zero. For events that took place after the last stimulus onset, we assumed that norm $\left(t_{\text {onset }}\right)=t_{r, n}+$ $t_{\text {onset }}-t_{a, n}$, with $t_{r, n}$ and $t_{a, n}$ being the onset times of the last stimulus in the reference session and Session A, respectively.

\section{Results and Discussion}

The tendency to initiate false alarms varied considerably over subjects, as can be concluded from the individual $\lambda$ s and $\mu$ s in Table 2. In SF sessions, 5 subjects did not respond at all, whereas the 2 most eager subjects responded 9 and 14 times, respectively. These 2 subjects were experienced panelists. In addition, closer inspection
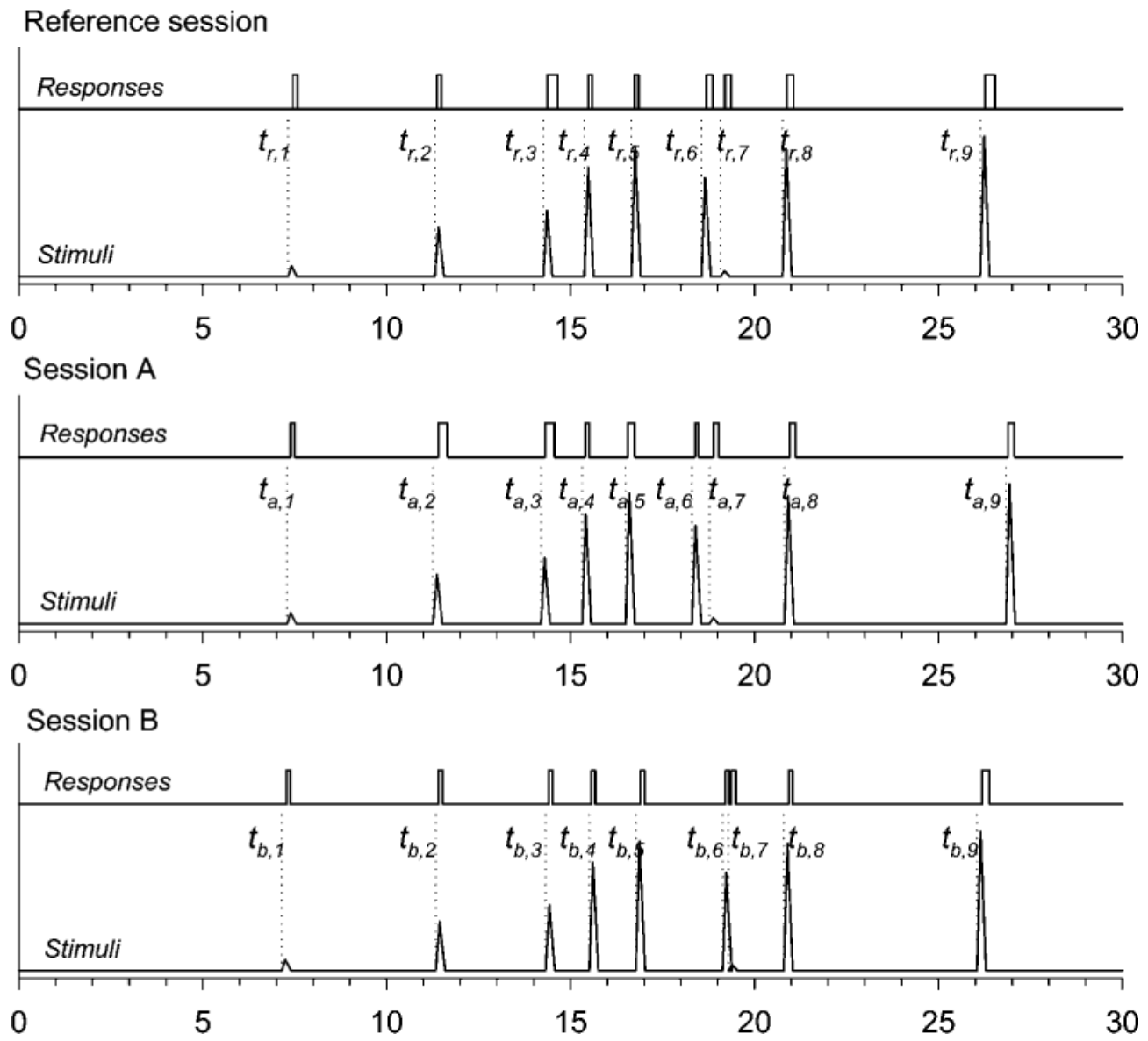

Figure 2. Example of normalization of sniffing response times. Stimulus retention times and the corresponding sniffing response times for nine odorants (1-9) in the reference session and in two sessions with slightly deviating retention times (Sessions A and B). The dotted lines indicate stimulus onset times. Response onset times and response offset times are normalized by setting stimulus onset times to the onset times of the same stimuli in the standard session and calculating response event times by linear interpolation (see the text). 
Table 2

Estimates of Onset Rates $(\hat{\lambda})$ and Offset Rates $(\hat{\mu})$ for Individual Subjects and the Panel, Based on Trimmed Stimulus-Present Sessions (False Alarms), Stimulus-Free Sessions (False Alarms), and Nontrimmed Stimulus-Present Sessions (All Responses)

\begin{tabular}{|c|c|c|c|c|c|c|c|c|c|}
\hline \multirow[b]{2}{*}{ Subject } & \multicolumn{3}{|c|}{$\begin{array}{c}\text { Trimmed Stimulus-Present } \\
\text { (Observation Window }=17.74 \mathrm{~min} \text { ) } \\
\end{array}$} & \multicolumn{3}{|c|}{$\begin{array}{c}\text { Stimulus-Free } \\
\text { (Observation Window }=30.00 \mathrm{~min}) \\
\end{array}$} & \multicolumn{3}{|c|}{$\begin{array}{c}\text { Stimulus-Present (nontrimmed) } \\
\text { (Observation Window }=30.00 \mathrm{~min})\end{array}$} \\
\hline & $\hat{\lambda}\left(\min ^{-1}\right)$ & $\hat{\mu}\left(\min ^{-1}\right)$ & No. Resp. & $\hat{\hat{\lambda}}\left(\min ^{-1}\right)$ & $\hat{\mu}\left(\min ^{-1}\right)$ & No. Resp. & $\hat{\hat{\lambda}}\left(\min ^{-1}\right)$ & $\hat{\mu}\left(\min ^{-1}\right)$ & No. Resp. \\
\hline 1 & 0.115 & 6.08 & 2 & 0.067 & 13.57 & 2 & 0.206 & 6.64 & 6 \\
\hline 2 & 0.000 & - & 0 & 0.306 & 15.15 & 9 & 0.101 & 12.28 & 3 \\
\hline 3 & 0.000 & - & 0 & 0.102 & 4.38 & 3 & 0.275 & 9.01 & 8 \\
\hline 4 & 0.305 & 3.66 & 5 & 0.252 & 3.22 & 7 & 0.456 & 3.29 & 12 \\
\hline 5 & 0.000 & - & 0 & 0.000 & - & 0 & 0.219 & 2.30 & 6 \\
\hline 6 & 0.056 & 45.45 & 1 & 0.000 & - & 0 & 0.184 & 1.77 & 5 \\
\hline 7 & 0.229 & 15.00 & 4 & 0.481 & 15.92 & 14 & 0.420 & 8.56 & 12 \\
\hline 8 & 0.377 & 1.12 & 5 & 0.331 & 1.37 & 8 & 0.459 & 1.22 & 10 \\
\hline 9 & 0.000 & - & 0 & 0.000 & - & 0 & 0.210 & 4.11 & 6 \\
\hline 10 & 0.173 & 7.55 & 3 & 0.000 & - & 0 & 0.387 & 7.09 & 11 \\
\hline 11 & 0.057 & 3.44 & 1 & 0.102 & 5.75 & 3 & 0.257 & 2.51 & 7 \\
\hline 12 & 0.000 & - & 0 & 0.033 & 11.03 & 1 & 0.139 & 3.32 & 4 \\
\hline 13 & 0.114 & 16.67 & 2 & 0.168 & 26.02 & 5 & 0.236 & 18.59 & 7 \\
\hline 14 & 0.127 & 1.03 & 2 & 0.000 & - & 0 & 0.378 & 0.61 & 7 \\
\hline 15 & 0.115 & 7.38 & 2 & 0.067 & 8.18 & 2 & 0.347 & 8.77 & 10 \\
\hline 16 & 0.113 & 19.67 & 2 & 0.067 & 39.74 & 2 & 0.349 & 7.61 & 10 \\
\hline Panel & 0.106 & 3.03 & 29 & 0.120 & 4.90 & 56 & 0.283 & 2.95 & 124 \\
\hline
\end{tabular}

Note-Panel parameters were estimated by concatenating the results of the 16 panelists, as if 1 person performed a session that was 16 times as long. No. Resp., number of responses.

of the FID readings of the respective sessions showed no evidence of irregularities that might have caused these outcomes. Therefore, all the data were used for the analyses.

Although the subjects clearly varied in the number of false alarms, they showed fairly consistent intraindividual response behavior over experimental sessions. In general, high responders in the SF sessions were also high responders in the SF windows of the SP sessions, and vice versa, low responders in the SF sessions generally showed low response behavior in the SF windows of the SP sessions. Accordingly, the subjects' numbers of responses correlated significantly between the two experimental conditions (Pearson $r=.52, p=.040$ ). Because responses in the SF sessions and in the trimmed sections of the SP sessions consisted of false alarms only, we attribute individual differences in onset rates to different decision criteria.

Colquhoun and Baddeley (1964) showed that after previous sessions with high stimulus probabilities, the initial decision criterion $\beta$ in the current session was lower than that after sessions with low stimulus probabilities. Furthermore, $\beta$ gradually increased during the current session. The authors argued that this was caused by unfulfilled expectations of stimulus probability. The increasing response conservatism that follows from the gradual increase of $\beta$ is seen as an adaptive response to the decreasing anticipated stimulus probability in order to optimize response behavior. In the present study, the stimulus density dropped from 9 per SP session to 0 per SF session. Because SF sessions were preceded by one to seven SP sessions and because the stimulus probability had been identical for all SP sessions, it is likely that the subjects had strong a priori expectations of stimulus probability when they embarked on the SF session. In contrast to the study by Colquhoun and Baddeley (1964), in which changes of stimulus probability could readily be attributed to experimental conditions, the subjects in the present study probably attributed the unexpected and complete absence of stimuli to their own inability to smell: Many subjects reported to be frustrated with their own smelling inability on completion of the SF session. Instead of increasing their $\beta$ in response to externally attributed changes in stimulus frequency, the subjects would have to decrease their $\beta$ if they wanted their response frequencies to remain at the level of previous sessions, in order to compensate for their alleged smelling inability. This would result in higher onset rates $\lambda$ in SF sessions than in trimmed SP sessions.

Whereas in SF vigilance conditions the response onset rate is primarily associated with the decision criterion $\beta$ the offset rate, being a measure of response length, does not have a direct counterpart in terms of SDT. A possible factor influencing the offset rate in the case of false alarms may be response confidence. Once an observer has initiated a response, he or she will continuously evaluate whether the stimulus is still present or even whether it has ever been present at all. Having lowered their decision criteria in response to an unexpected absence of stimuli in SF sessions, subjects' response confidence may also drop. As a result, they would terminate responses (being false alarms) earlier in SF sessions than in trimmed SP sessions, resulting in higher offset rates in SF sessions than in trimmed SP sessions. In summary, both $\lambda$ and $\mu$ are expected to be smaller in trimmed SP sessions than in SF sessions, implying that the average latency and the average response length are expected to be larger in trimmed SP sessions than in SF sessions. Furthermore, the presence of stimuli in the nontrimmed 
SP session is expected to increase the average response length (lower $\mu$ ) and to decrease the average latency between responses (higher $\lambda$ ), in comparison with the trimmed session.

We tested individual values of $\lambda$ and $\mu$ for these hypothesized effects of stimulus condition (trimmed SP, SF, and nontrimmed SP). To obtain metrically comparable measures suitable for an analysis of variance (ANOVA), reciprocals of all observed individual $\lambda$ s and $\mu$ s were calculated, yielding measures that reflected response latency and response length, respectively. Some subjects failed to generate false alarms in SF or trimmed SP sessions and, hence, did not produce estimates of response length (Table 2). As a consequence, effects of stimulus condition were analyzed by a repeated measures ANOVA on complete data sets of the remaining 8 subjects. Where necessary, test results were corrected for deviations from sphericity by multiplying the numerator and denominator degrees of freedom with Greenhouse-Geisser's $\varepsilon$.

Significant condition effects were observed for both response latency $[F(2,14)=6.04, p=.015]$ and response length $[F(2,14)=4.40, p=.037]$. As was expected, panel values of $\lambda$ and $\mu$ were lower for trimmed SP sessions than for SF sessions $(\lambda=0.106$ vs. 0.120 and $\mu=3.03$ vs. 4.90 , respectively). However, contrasts comparing the reciprocals of $\lambda$ and $\mu$ between trimmed SP sessions and SF sessions were not significant for latency $[F(1,7)=0.225, p=.650]$ or for length $[F(1,7)=$ $4.39, p=.074]$. Tests of separate contrasts showed that the significant overall test results could be attributed to the difference between the effects of trimmed SP and nontrimmed SP sessions on latency [the corresponding $\lambda$ s are 0.106 and 0.283 , respectively; $[F(1,7)=10.12, p=$ $.015]$ and the difference between the effects of SF and nontrimmed SP sessions on response length [the corresponding $\mu$ s are 4.90 and 2.95 , respectively; $F(1,7)=$ $6.53, p=.038]$. Obviously, these effects are caused by responses to stimuli in the nontrimmed data.

It should be realized that the results of the repeated measures ANOVA do not provide the optimal conditions for demonstrating a differential influence of stimulus condition on QST noise models. Obviously, excluding 8 out of 16 subjects from the analysis has reduced the power of the test considerably. Also, the exclusion of nonresponding panelists from the repeated measures analysis causes a systematical underestimation of the average panel latency. A subject that generates zero responses during an SF period contributes as much to the estimate of panel QST parameters as a subject that responds at least once. Therefore, statistical tests of effects of stimulus presence on empirically obtained QST model parameters are poor indicators of the effects on the sensitivity of QST tests of DFs.

The DFs of the 16 panelists in the SP session are shown in Figure 3A, along with the stimulus onset times of the nine stimuli. The corresponding DFs in the SF session are shown in Figure 3B. Simulations of sessions based on $\hat{\lambda}$ and $\hat{\mu}$ from trimmed SP sessions and SF sessions are shown in Figures 3C and 3D, respectively. On visual inspection, these simulation results are very similar to the empirical results from the SF session in Figure $3 \mathrm{~B}$, regardless of the stimulus condition in which the parameters were estimated.

The SF session yields a maximum DF of 3 . Hence, according to the rule of thumb used in the DF method, DFs above 3 in the SP session are considered stimulus detections. Thus, six stimuli are detected-that is, Stimuli 1, $3,4,5,7$, and 8 .

The observed $T_{k}$ and the estimates $\hat{T}_{k}$ for trimmed SP and SF sessions are shown in Table 3. Observed $T_{k} \mathrm{~s}$ exceed QST $\hat{T}_{k}$ s estimates at all coincidence levels $(k>0)$ and for parameter estimates from both SF and trimmedSP sessions. Surely, it would be premature to conclude from these results that coinciding responses at all $k>0$ indicate stimulus detections. Although $\hat{T}_{k}$ can now be calculated from $\hat{\lambda}$ and $\hat{\mu}$ (Equations 6 and 7), insight into the distribution of $\hat{T}_{k}$ is needed to enable testing of empirical $T_{k}$ against the null-hypothesis that no stimuli are present. In the next section, we present a heuristic that estimates $\hat{T}_{k}$ variances from $\hat{\lambda}$ and $\hat{\mu}$. These variances will be used to test the significance of observed $T_{k}$ and of single sniffing peaks.

\section{DERIVING A HEURISTIC TO CALCULATE $T_{k}$ VARIANCE}

Although empirical results may be used to estimate critical values of response coincidence probabilities, this would require costly replications of sniffing sessions, very likely to the detriment of subjects' motivation. Therefore, we have derived a heuristic to calculate $\operatorname{Var}\left(\hat{T}_{k}\right)$ from $\hat{T}_{k}$, $\hat{\lambda}$, and $\hat{\mu}$. These variances are used to estimate critical values for $\hat{T}_{k}$. SF sniffing sessions are simulated assuming exponentially distributed onset and offset times. By systematically varying onset rates, offset rates, and session lengths, results can be used to derive the model relating $\hat{T}_{k}$ to $\operatorname{Var}\left(\hat{T}_{k}\right)$. In this section, we will discuss the routines and derive the heuristic. Subsequently, we will propose a post hoc analysis that tests whether individual sniffing peaks are above noise level.

\section{Method}

Simulation of sniffing data. Sniffing experiments were simulated using uniformly distributed random deviates $u(0 \leq u<1)$ generated by the Ran 3 routine (Press, Flannery, Teukolsky, \& Vetterling, 1989). To obtain exponentially distributed latencies, uniform deviates $u_{1}$ were transformed by

$$
\text { latency }=-\ln \left(1-u_{1}\right) / \lambda \text {, }
$$

where $\lambda$ is the onset rate. Similarly, exponentially distributed response lengths were calculated from uniform deviates $u_{2}$ by

$$
\text { length }=-\ln \left(1-u_{2}\right) / \mu \text {, }
$$

where $\mu$ is the offset rate. In a simulated session, initial simulated latencies all start at $t=0$, which systematically lowers $P_{k}$ values during the first minutes of a ses- 

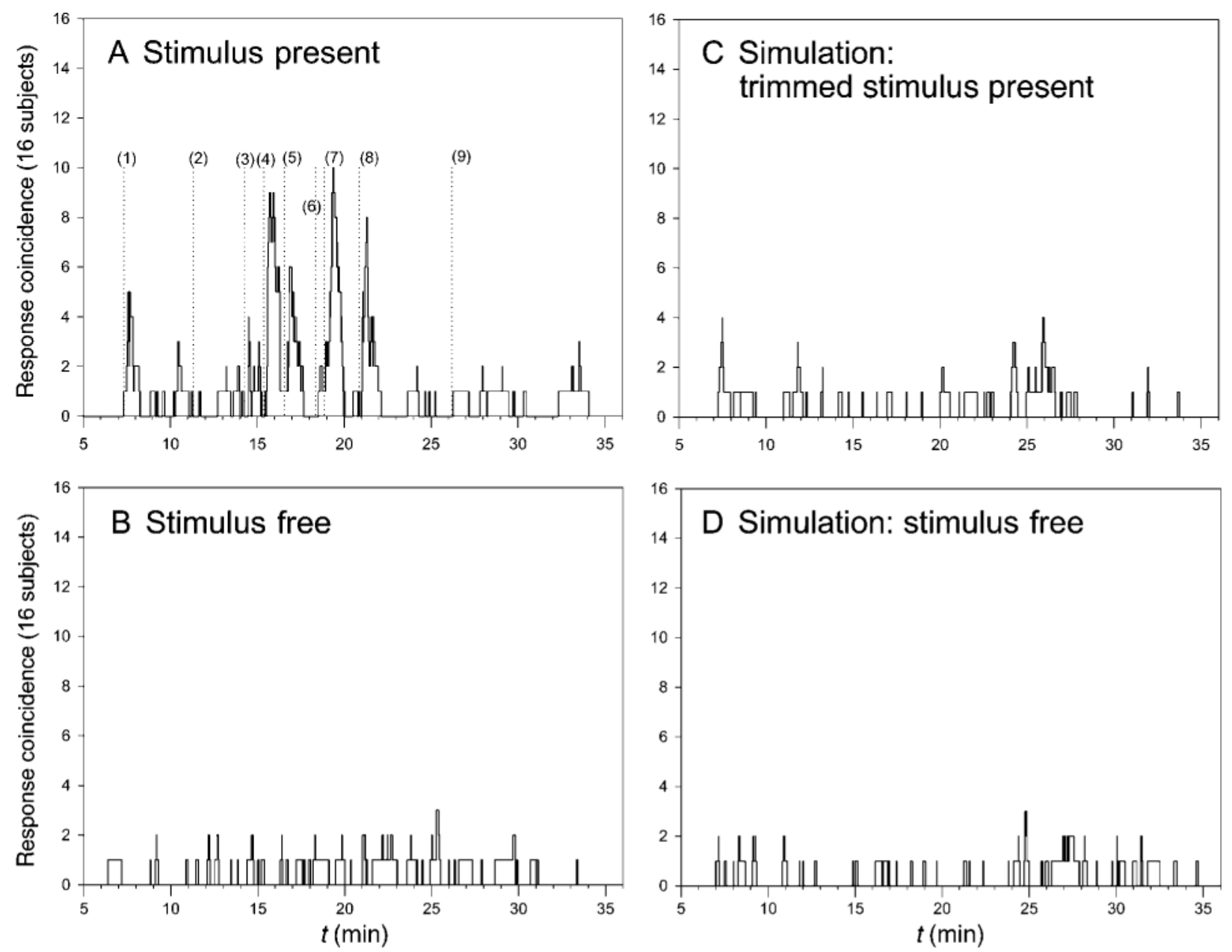

Figure 3. Response coincidences of 16 subjects plotted against experiment time. (A) Empirical results of the stimulus-present condition; stimulus onset times are indicated by dotted lines. Index numbers correspond with peak numbers in Table 1. Individual responses were normalized before calculation of response coincidences. Therefore, the times used on the abscissa reflect stimulus events in the reference session (see the text). (B) Empirical results of the stimulus-free condition. (C) Simulated results: Example of a coincidence plot generated using $\hat{\lambda}$ and $\hat{\mu}$ values calculated from the stimulus-free windows in stimulus-present sessions. (D) Simulated results: example of a coincidence plot generated with $\hat{\lambda}$ and $\hat{\mu}$ values calculated from the results in stimulus-free sessions.

sion. Consequently, we simulated 120-min sessions, of which only responses given during the final 60 min were used for calculation of $P_{k}$. All algorithms were written as Pascal routines embedded in a Delphi 4 environment (Borland Software Corporation, 1998).

Results from multiple simulated sessions may be used to calculate $P_{k}$ and $\operatorname{Var}\left(P_{k}\right)$. Because the QST model and the simulations both assume exponential decay functions for onset and offset times, simulated $P_{k}$ s are best predicted by model estimates of $P_{k}$, using the same $\lambda$ and $\mu$ as those in Equations 6 and 7. Therefore, we first crossvalidated simulated $P_{k}$ with $P_{k}$ predicted by the QST model in Simulation 1. In this simulation, $P_{k}$ s were obtained by simulating "SF" sessions, using the values of $\lambda$ and $\mu$ specified in Table 4 . These onset and offset rates represent a range of realistic values (see the empirical section). The simulated panel size was 20 . One thousand sessions were simulated for each of the 25 possible combinations of $\lambda$ and $\mu$. Subsequently, the resulting averaged $P_{k}$ s were cross-validated with modeled $P_{k}$ s (Equation 6).

Estimation of $\boldsymbol{P}_{\boldsymbol{k}}$ variances supplementing model estimates of $\boldsymbol{P}_{\boldsymbol{k}}$. As is implied by the central limit theorem, raising response incidences in the time window used to estimate $\lambda$ and $\mu$ results in narrower probability distributions for $\hat{\lambda}$ and $\hat{\mu}$ and, consequently, in narrower probability distributions for $\hat{P}_{k}$. Therefore, the expected number of responses per time unit should be considered for the calculation of $\operatorname{Var}\left(\hat{P}_{k}\right)$. If the number of responses per time unit increases (when $\lambda$ increases and/or $\mu$ decreases), $\operatorname{Var}\left(\hat{P}_{k}\right)$ will decrease. To estimate the number of responses per time unit, we assess the number of completed responses that fit one time unit. The time needed to complete a response is defined as the average time be- 
Table 3

Modeled Cumulative Time at Response Coincidence Level $k\left(T_{k}\right)$, the Respective Critical Values of $\hat{\boldsymbol{T}}_{\boldsymbol{k}}$ at Significance Levels .05 and .01, and Observed Times $T_{k}$ at Specified Coincidence Levels

\begin{tabular}{|c|c|c|c|c|c|c|c|}
\hline \multirow[b]{2}{*}{$\begin{array}{l}\text { Coincidence } \\
\text { Level }(k)\end{array}$} & \multirow{2}{*}{$\begin{array}{c}T_{k}(\min ) \text { Observed } \\
\text { in Nontrimmed } \\
\text { Stimulus Present } \\
\text { at Session }\end{array}$} & \multicolumn{3}{|c|}{$\begin{array}{c}\text { Stimulus Present (Trimmed); } \\
\hat{\lambda}=0.106\left(\mathrm{~min}^{-1}\right), \hat{\mu}=3.03\left(\mathrm{~min}^{-1}\right)\end{array}$} & \multicolumn{3}{|c|}{$\begin{array}{c}\text { Stimulus Free; } \\
\hat{\lambda}=0.120\left(\min ^{-1}\right), \hat{\mu}=4.9\left(\mathrm{~min}^{-1}\right)\end{array}$} \\
\hline & & $\begin{array}{l}\text { Model } \hat{T}_{k} \\
\text { (min:sec) }\end{array}$ & $\begin{array}{c}\text { Upper Critical } \hat{T}_{k} \\
\text { at } \alpha=.05(\mathrm{~min}: \mathrm{sec})\end{array}$ & $\begin{array}{c}\text { Upper Critical } \hat{T}_{k} \\
\text { at } \alpha=.01(\mathrm{~min}: \mathrm{sec})\end{array}$ & $\begin{array}{l}\text { Model } \hat{T}_{k} \\
\text { (min:sec) }\end{array}$ & $\begin{array}{c}\text { Upper Critical } \hat{T}_{k} \\
\text { at } \alpha=.05 \text { (min:sec) }\end{array}$ & $\begin{array}{c}\text { Upper Critical } \hat{T}_{k} \\
\text { at } \alpha=.01(\mathrm{~min}: \mathrm{sec})\end{array}$ \\
\hline 0 & $15: 08.2$ & $17: 18.3$ & - & - & $20: 22.2$ & - & - \\
\hline 1 & $09: 27.9$ & $09: 41.2$ & $14: 26.3$ & $16: 55.5$ & $07: 58.9$ & $11: 19.4$ & $13: 01.4$ \\
\hline 2 & $02: 00.9$ & $02: 32.5$ & $04: 18.4$ & $05: 18.1$ & $01: 28.0$ & $02: 31.0$ & 03:06.9 \\
\hline 3 & $01: 01.4$ & $00: 24.9$ & $01: 02.2$ & $01: 28.8$ & $00: 10.1$ & $00: 28.9^{*}$ & $00: 43.7 * *$ \\
\hline 4 & $00: 36.4$ & $00: 02.8$ & $00: 14.4^{*}$ & $00: 23.9^{* *}$ & $00: 00.8$ & $00: 05.7^{*}$ & $00: 10.4 * *$ \\
\hline 5 & $00: 32.6$ & $00: 00.2$ & $00: 03.4 *$ & $00: 06.4 * *$ & 00:00.0 & $00: 01.2 *$ & $00: 02.4 * *$ \\
\hline 6 & $00: 26.7$ & 00:00.0 & $00: 00.8^{*}$ & $00: 01.6^{* *}$ & $00: 00.0$ & $00: 00.2^{*}$ & $00: 00.5^{* *}$ \\
\hline 7 & $00: 12.6$ & 00:00.0 & $00: 00.2 *$ & $00: 00.4 * *$ & $00: 00.0$ & $00: 00.0 *$ & $00: 00.1^{* *}$ \\
\hline 8 & $00: 18.2$ & $00: 00.0$ & $00: 00.0 *$ & $00: 00.1 * *$ & $00: 00.0$ & $00: 00.0^{*}$ & $00: 00.0 * *$ \\
\hline 9 & $00: 14.3$ & $00: 00.0$ & $00: 00.0 *$ & $00: 00.0 * *$ & $00: 00.0$ & $00: 00.0^{*}$ & $00: 00.0^{* *}$ \\
\hline 10 & $00: 01.0$ & $00: 00.0$ & $00: 00.0^{*}$ & $00: 00.0 * *$ & 00:00.0 & $00: 00.0^{*}$ & $00: 00.0^{* *}$ \\
\hline 11 & - & 00:00.0 & 00:00.0 & 00:00.0 & 00:00.0 & $00: 00.0$ & $00: 00.0$ \\
\hline 12 & - & 00:00.0 & 00:00.0 & 00:00.0 & 00:00.0 & 00:00.0 & 00:00.0 \\
\hline
\end{tabular}

Note- Modeled $\hat{T}_{k}$ and respective upper critical values are shown for model parameters estimated from stimulus-free and trimmed stimuluspresent sessions. $\quad * p<.05 . \quad * * p<.01$.

fore responding $(1 / \lambda)$ plus the average response length $(1 / \mu)$. The number of completed responses per time unit is the reciprocal thereof:

$$
n_{\text {expected }}=\frac{1}{\lambda^{-1}+\mu^{-1}}=\frac{(\lambda \cdot \mu)}{(\lambda+\mu)} .
$$

Furthermore, because $P_{k}$ s are proportion scores, their distributions will have a positive skew at sufficiently small $P_{k}$, approximating a gamma distribution (see the Appendix). Being approximately gamma distributed, $P_{k} \mathrm{~s}$ relate linearly to $\operatorname{Var}\left(P_{k}\right)$ (Winer, Brown, \& Michels, 1991). Later in this simulation section, we will determine up to which $P_{k}$ values this linear relation is valid. As for now, we assume the model to be linear. The coefficient $\lambda / \mu$, expressed as the parameter $\rho$, is a measure for the relative length of a response with respect to its incidence. Relatively long responses result in larger variances. Therefore, $\rho$ is expected to relate positively to $\operatorname{Var}\left(\hat{P}_{k}\right)$. The coincidence level $k$ relates inversely to $\operatorname{Var}\left(\hat{P}_{k}\right)$ because individual responses do not coincide for the full length of the responses but, rather, overlap par- tially, causing the length of coincidences to decrease with increasing $k$. Summarizing, in its simplest linear form, a heuristic relating $\operatorname{Var}\left(\hat{P}_{k}\right)$ to $\hat{P}_{k}$ would read

$$
\operatorname{Var}\left(\hat{P}_{k}\right)=c \cdot \frac{\rho \cdot \hat{P}_{k}}{k \cdot n_{\text {expected }}}, \quad(k>0),
$$

where $\operatorname{Var}\left(P_{k}\right)$ is the variance of the proportional length of responses at coincidence $k, c$ is an arbitrary constant, and $\rho=\lambda / \mu$. $\operatorname{Var}\left(\hat{T}_{k}\right)$ can be obtained by multiplying $\operatorname{Var}\left(\hat{P}_{k}\right)$ with the session length (see also Equation 7). This concise model does not define variances at $k=0$. Yet this will not interfere with testing empirical $T_{k}$ in detection experiments, because coincidence levels of 0 are irrelevant in that context.

To test whether the assumptions underlying the heuristic are plausible and to determine the size of the constant $c$, four extra series of experiments were simulated in which the parameters $n_{\text {expected }}, l_{\text {session }}$, and $\rho$ were systematically varied. The designs of these four series are shown in Table 4. In Simulation 2, $n_{\text {expected }}$ values were manipulated by varying onset and offset rates, maintain-

Table 4

Simulated Experiments: Simulation Experiment Numbers (as Referred to in the Text), Onset Rates $(\lambda)$, Offset Rates $(\mu)$, the Ratio of $\lambda$ and $\mu(\rho)$, Length of the Data Collection Window $\left(l_{\text {session }}\right)$, the Number of Replications of Each Simulation Experiment That Were Used to Calculate

\begin{tabular}{|c|c|c|c|c|c|c|c|}
\hline $\begin{array}{l}\text { Simulation } \\
\text { Number }\end{array}$ & $\begin{array}{c}\lambda \\
\left(\min ^{-1}\right)\end{array}$ & $\begin{array}{c}\mu \\
\left(\min ^{-1}\right)\end{array}$ & $\rho$ & & $\begin{array}{l}l_{\text {session }} \\
(\mathrm{min})\end{array}$ & $\begin{array}{c}\text { Number of } \\
\text { Replications }\end{array}$ & $M$ \\
\hline 1 & $0.01,0.05,0.10,0.15,0.25$ & $1,2,3,6,10$ & variable & 60 & & 1,000 & 20 \\
\hline 2 & $0.01^{\mathrm{a}}, 0.04^{\mathrm{b}}, 0.16^{\mathrm{c}}, 0.64^{\mathrm{d}}$ & $0.5^{\mathrm{a}}, 2^{\mathrm{b}}, 8^{\mathrm{c}}, 32^{\mathrm{d}}$ & 0.02 & 60 & & 100 & 20 \\
\hline 3 & $0.01,0.05,0.10,0.15,0.25$ & $1,2,3,6,10$ & variable & 60 & & 100 & 20 \\
\hline 4 & $0.01,0.05,0.10,0.15,0.25$ & $1,2,3,6,10$ & variable & 60 & & 100 & 10 \\
\hline 5 & 0.01 & 0.5 & 0.02 & 60 & $40,960,3,840$ & 100 & 20 \\
\hline
\end{tabular}
Averages of $\hat{T}_{k}$ and $\operatorname{Var}\left(\hat{T}_{k}\right)$, and the Number of Panelists That Were Simulated $(M)$

Note-All simulated experimental windows from which data were used were preceded by 60 -min windows from which data were discarded. a,b,c,dThe parameters $\lambda$ and $\mu$ were chosen in a way that $\rho$ always equalled 0.02 . Hence, four simulations ( $\mathrm{a}, \mathrm{b}, \mathrm{c}$, and d) were performed by combining $\lambda$ s and $\mu$ s that shared identical index letters. 

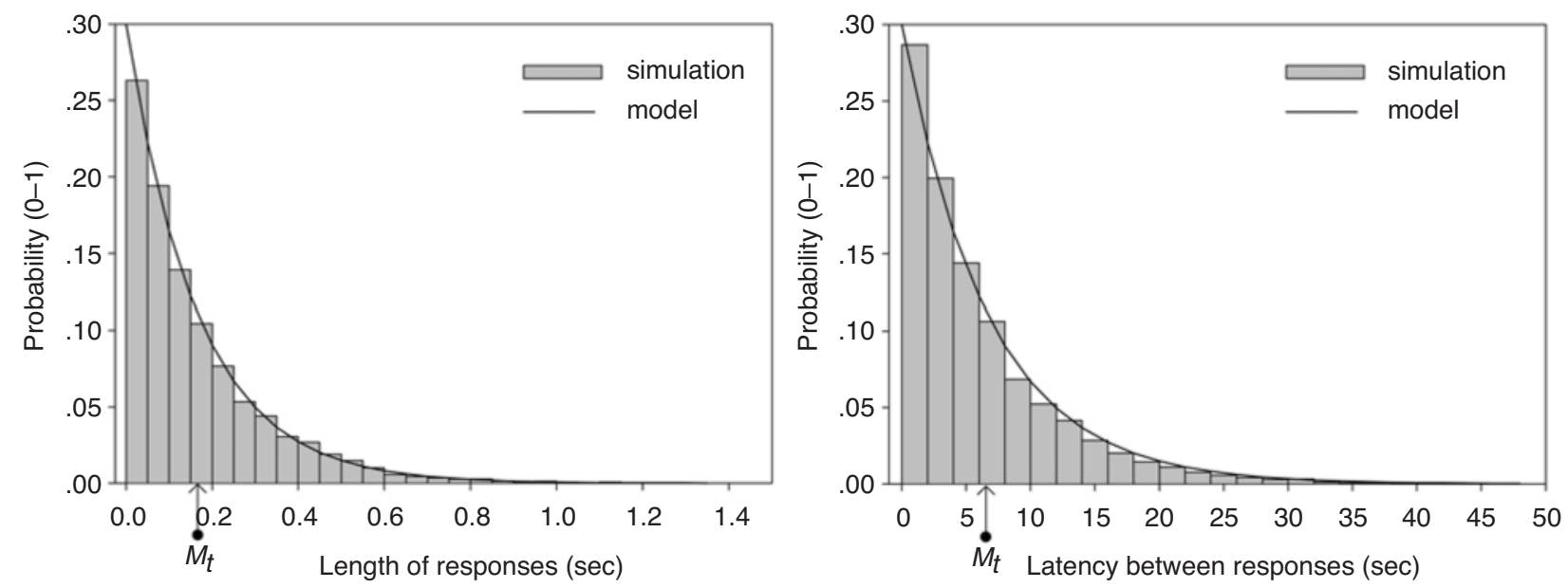

Figure 4. Proportional densities of response length (left panel) and response latencies (right panel) as calculated by Equation 6 with stimulus onset rate $\lambda=0.15\left(\mathrm{~min}^{-1}\right)$ and stimulus offset rate $\mu=6\left(\mathrm{~min}^{-1}\right)$ for 1,000 simulations of 1 subject. Modeled probabilities are plotted in the same graph, coinciding perfectly with calculated probabilities. Average $t(M t)$ equals the reciprocal of the model parameters.

ing a constant $\rho$. In Simulation 3, $\rho$ was manipulated by using 25 factorial combinations of five different $\lambda$ s and five different $\mu \mathrm{s}$. As a control for the influence of panel size, we ran the same series of 25 experiments again for a different number of subjects (Simulation 4). Length of experimental sessions was manipulated in a series of four experiments with varying session lengths (Simulation 5). Each of these simulations was conducted 100 times. $\hat{P}_{k}$ and $\operatorname{Var}\left(\hat{P}_{k}\right)$ were calculated for each $M, \lambda, \mu$, and $l_{\text {session }}$ condition. $\operatorname{Var}\left(P_{k}\right)_{\text {simulation }}$ was then evaluated against $\operatorname{Var}\left(\hat{P}_{k}\right)$ to estimate the constant $c$ in Equation 13.

Testing observed $T_{k}$ and post hoc testing of individual sniffing peaks. If panelists observe stimuli, their responses will increase in number and in length, which will raise the probability of responses to coincide. Consequently, the cumulated time at coincidence levels $k>1$ will increase accordingly. Because $\hat{T}_{k}$ and $\operatorname{Var}\left(\hat{T}_{k}\right)$ are estimated from responses given when no stimuli were presented, these values can be used to test whether the probability of observed $T_{k}$ is above chance level. Upper critical values of $T_{k}\left(T_{k}^{\prime}\right)$ were calculated for all $k \mathrm{~s}$ at significance levels .05 and .01 . Given some $\hat{\lambda}$ and $\hat{\mu}, \hat{T}_{k} \mathrm{~s}$ close to zero have skewed distributions, because zero constitutes a lower bound to observable $T_{k}$ values. Therefore, it should be noted that simply assuming $T_{k}$ to be Gaussian distributed in order to calculate critical values of $T_{k}$ might seriously threaten test reliability (see the Appendix). Instead, we assumed gamma-distributed $T_{k}$ with variance $\operatorname{Var}\left(\hat{T}_{k}\right)$ and average $\hat{T}_{k}$.

All the tests were one-sided because relevant $T_{k} \mathrm{~s}$ are expected to increase when stimuli are detected. However, because

$$
\sum_{k=0}^{M} T_{k}=l_{\text {session }},
$$

an increased response incidence can reduce $T_{k}$ at low coincidence levels (i.e., $k=0,1$ ) to the extent that these would be significant if two-sided tests were used. Because we were interested mainly in increases of response coincidences, and because the decrease of $T_{k}$ at low $k$ can be regarded as a side effect of the increases we were interested in, we restricted ourselves to the use of upper critical levels for $T_{k}$.

If $T_{k}$ were independent and if an aggregate test of $T_{k}$ was to be performed at some significance level $\alpha$, it would be advisable to adjust the significance levels for single tests at each level of $k$ to $1-(1-\alpha)^{1 / k}$. This would result in strict significance levels per level of $k$ in order to preserve the desired overall level of significance. However, as was discussed earlier, $T_{k}$ is linearly dependent. Furthermore, $T_{k}$ can only be larger than zero if $T_{k-1}>0$. Hence, adjustment of the $\alpha$ levels is undesirable. We will test at $\alpha=.01$ and .05 .

To identify which sniffing peaks contributed to significant $T_{k}$ values, we performed post hoc tests at coincidence levels that produced significant $T_{k}$. Under the null hypothesis that all responses are false alarms, it is assumed that sniffing peaks may occur at any point in time at a constant probability. Each partial $T_{k}$ associated with a single sniffing peak $\left(\mathrm{T}_{k, i}, i\right.$ being the peak index) can then be tested against a critical value that is based on an observation window of the length $l_{\text {session }} / N_{k}$, with $N_{k}$ being the number of peaks that contain coinciding responses at level $k$. For a given significance level $\alpha$, the critical response length summarized over all peaks in a session $T_{k}^{\prime}$ is the $T_{k}$ value at which the corresponding cumulative probability distribution equals $1-\alpha$. Accordingly, $T_{k}^{\prime}$ can be represented as a linear function of the estimated standard deviation of $T_{k}$ by $\hat{T}_{k}+a \cdot S D\left(\hat{T}_{k}\right)[a>0]$. 

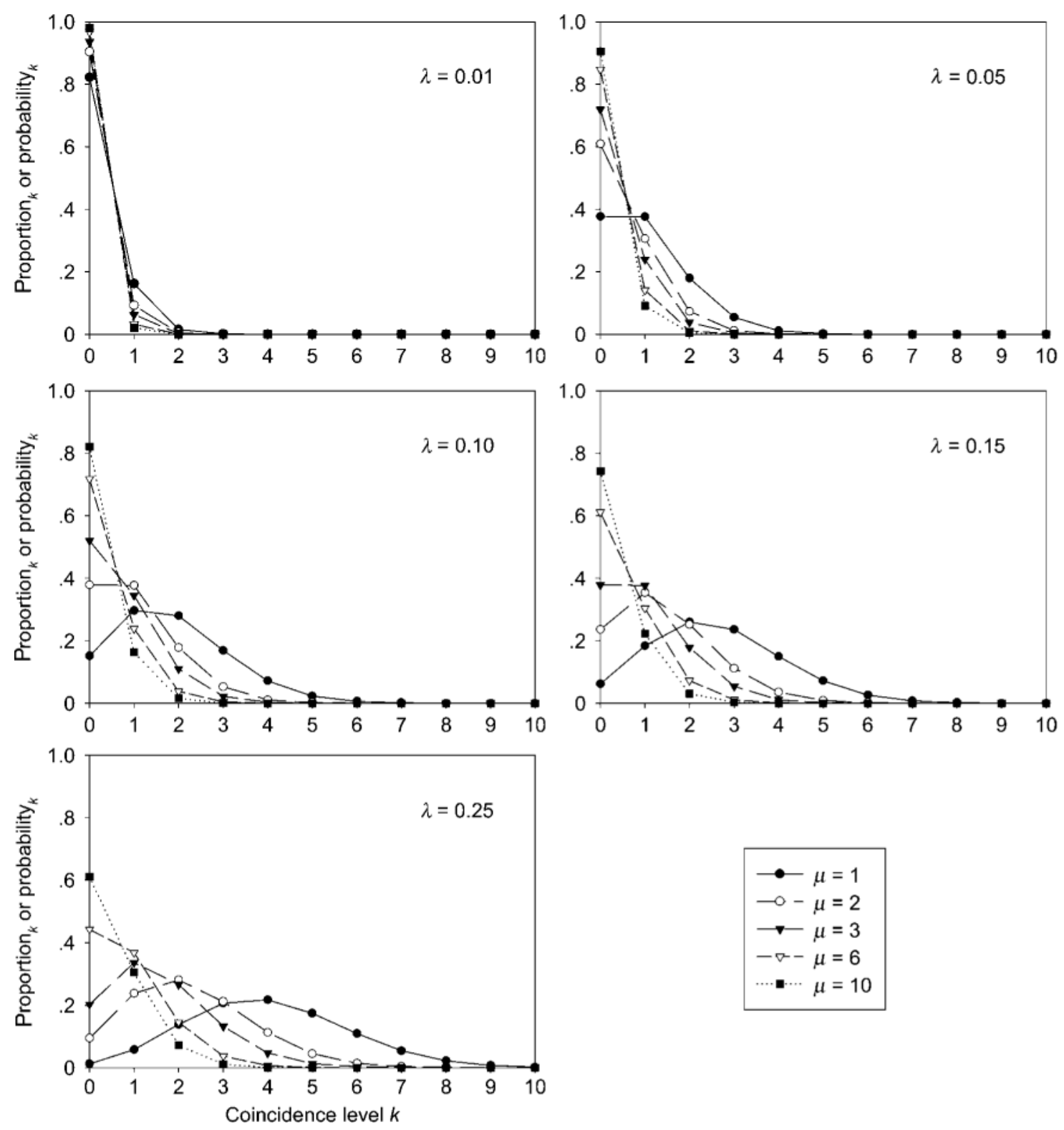

$$
\begin{aligned}
& \longrightarrow \mu=1 \\
& \longrightarrow-\mu=2 \\
& \longrightarrow-\mu=3 \\
& \longrightarrow-\mu=6 \\
& \cdots \cdots=10
\end{aligned}
$$

Figure 5. Response probabilities (modeled) and proportions of the total experimental session length (simulations) as functions of coincidence level $k$ and specified for different onset $(\lambda)$ and offset $(\mu)$ rates. Panel size is 20 subjects. Data points represent averages over 1,000 simulated experiments.

Per single-peak observation window, the expected $\hat{T}_{k, i}$ equals $\hat{T}_{k} / N_{k}$, and given the linear dependency of $\operatorname{Var}\left(\hat{T}_{k}\right)$ on $\hat{T}_{k}$, $\operatorname{Var}\left(\hat{T}_{k, i}\right)$ equals $\operatorname{Var}\left(\hat{T}_{k}\right) / N_{k}$. From this, we calculated single-peak critical values of $T_{k, i}^{\prime}$ by

$$
T_{k, i}^{\prime}=\frac{\hat{T}_{k}}{N_{k}}+a \cdot \sqrt{\frac{\operatorname{Var}\left(\hat{T}_{k}\right)}{N_{k}}}=\frac{\hat{T}_{k}}{N_{k}}+\frac{a \cdot S D\left(\hat{T}_{k}\right)}{\sqrt{N_{k}}},
$$

with $k$ being the coincidence level, $i$ the peak index, and $N_{k}$ the number of peaks containing coincidences at level $k$. The constant $a$ is derived from the global critical value $T_{k}^{\prime}$ and depends on the chosen $\alpha$.

\section{Results and Discussion}

The variance heuristic. Simulated $\hat{T}_{k}$ s were obtained per session and averaged over replications. Figure 4 shows that the corresponding $\hat{P}_{k}$ s obtained in Simulation 1 perfectly fitted probabilities predicted by the QST model. For the applied range of $\lambda$ and $\mu$ values, these measures proved to be identical up to the third decimal. There- 
fore, averages of the simulated $\hat{P}_{k}$ s are considered unbiased estimators of model probabilities. Consequently, we will use variances of the simulated $\hat{P}_{k}$ to optimize the heuristic for variance calculation.

In addition, simulated response lengths and latencies of Simulation 1 were analyzed to see whether these corresponded with the $\mu$ and $\lambda$ used in the simulation algorithm. For all the values of $\mu$ and $\lambda$ used, the average lengths and the average latencies indeed corresponded with their respective $\mu$ s and $\lambda_{\mathrm{s}}$ according to the model specified in Equations 8 and 9. For example, for simulations with $\mu=6$ and $\lambda=0.15$, the resulting average response length was $0.167 \mathrm{~min}$ and the average latency was $6.67 \mathrm{~min}$, resulting in $\hat{\mu}=5.99$ and $\hat{\lambda}=0.15$, respectively (Figure 5). Distributions of simulated response length and response latency also fitted their respective exponential probability distributions for all $\lambda-\mu$ combinations. This is illustrated in Figure 4 for $\lambda=0.15$ and $\mu=6$ with $n_{\text {simulations }}=1,000$.

If the estimated variance $\operatorname{Var}\left(\hat{P}_{k}\right)_{\text {model }}$ equals the simulated variance $\operatorname{Var}\left(P_{k}\right)_{\text {simulation, }}$, their quotient will equal 1 . The observed ratio of $\operatorname{Var}\left(\hat{P}_{k}\right)_{\text {model }}$ by $\operatorname{Var}\left(P_{k}\right)_{\text {sim- }}$ ulation produces an approximately horizontal line for $P_{k}$ values ranging from .001 up to .1 (Figure 6). For sniffing sessions of $60 \mathrm{~min}$, this $P_{k}$ range represents a $T_{k}$ of 3.6-360 sec, which largely covers empirically encountered $T_{k}$ at $k$ levels well above zero. Therefore, we optimized the value of $c$ in the heuristic in Equation 13 by fitting $\operatorname{Var}\left(\hat{P}_{k}\right)_{\text {model }} / \operatorname{Var}\left(P_{k}\right)_{\text {simulation }}$ ratios to a geometrical average variance ratio of 1 for $.001<P_{k}<.1$ by the least sum of squares method, using logarithms of variance ratios. The use of the geometric mean gives the same weights to identical proportional differences between ratios, either above or below the center score. This analysis resulted in an optimal fit for $c$ equal to 2.14 . After solving for $n_{\text {expected }}$ (Equation 12) and $\rho=\lambda / \mu$, Equation 13 then results in

$$
\operatorname{Var}\left(\hat{P}_{k}\right)=\frac{2.14(\lambda+\mu)}{\lambda \cdot \mu^{2}} \cdot \hat{P}_{k}, \quad(k>0) .
$$

At $P_{k}$ values above .1 , ratios of variances rapidly increase, indicating that the heuristic tends to overestimate variances for these $P_{k}$ values. In 60-min SF sessions, these $P_{k}$ values would represent a $T_{k}$ of $6 \mathrm{~min}$ and more. Such $T_{k}$ values are found only at $k=0$ or $k=1$, due to the generally frequent occasions on which 0 or 1 panelist is responding. Because we assess detection at values of $\mathrm{k}$ well above zero, $P_{k}$ values above 11 will not be relevant for our decisions on noise levels. At $P_{k}$ s below .001 the variances appear to be slightly overestimated by the heuristic. This causes slightly more conservative testing at these $P_{k}$ values. Variance ratios at low $P_{k}$ s show larger variability than do ratios at high $P_{k}$ s (Figure 6). This is due to the small number of observations involved in the calculation of simulated variances. Similarly, simulations involving 10 subjects (Figure 6, Simulation 4) pro-

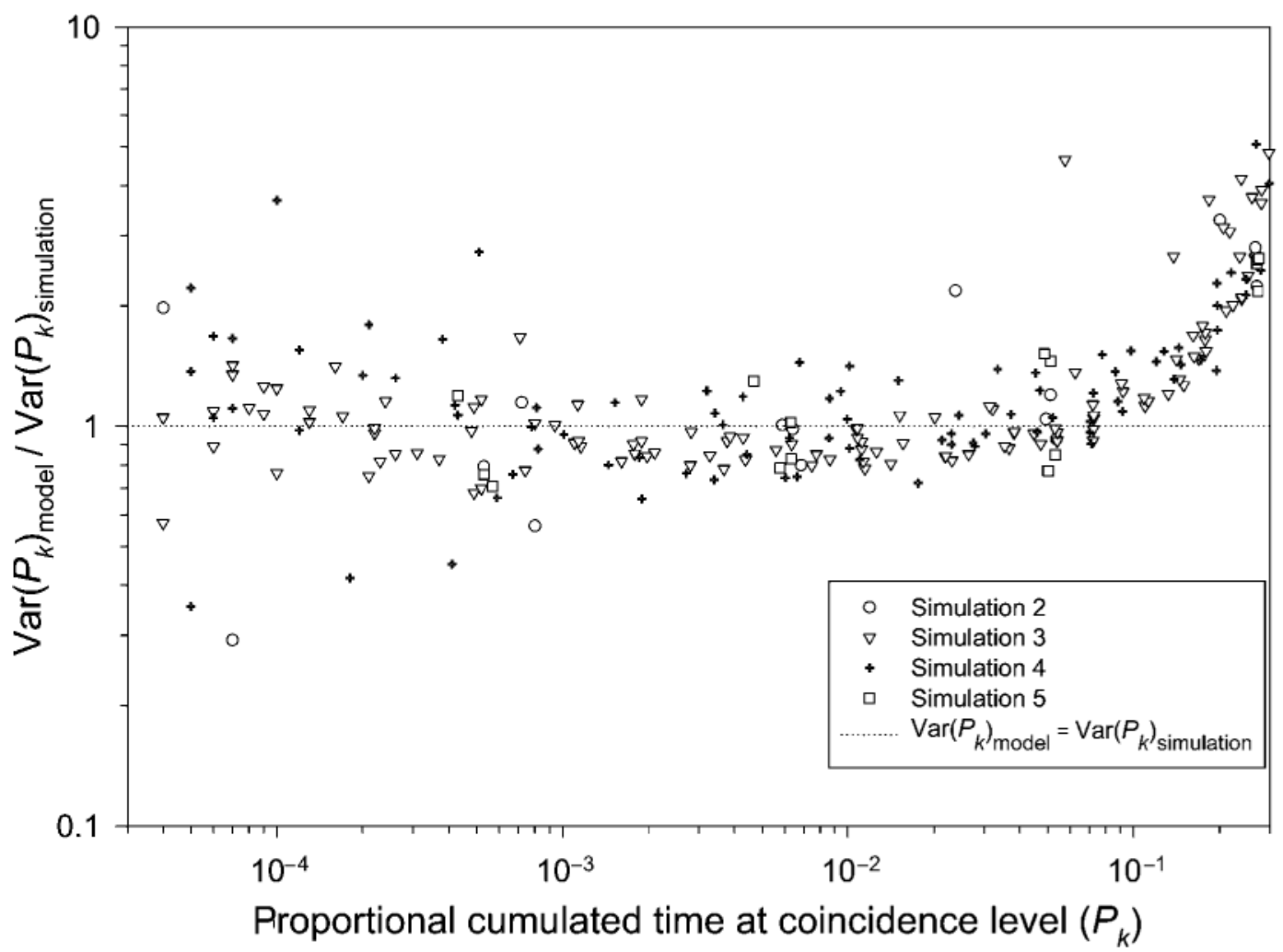

Figure 6. Ratios of modeled $P_{k}$ variance by simulated $P_{k}$ variance as a function of $\boldsymbol{P}_{k}$. Variance estimates are calculated by the heuristic discussed in the text (Equation 15). 
duce a larger variability of variance ratios than do those involving 20 subjects when the same parameter settings are employed (Figure 6, Simulation 3). Again, this is due to fewer observations in simulations of 10 subjects. However, panel size per se does not lead to different variance estimates. This can be concluded from the fact that variances for panel sizes 10 and 20 produce distributions of variance ratios that center on the same optimal variance ratio of 1 (Figure 6). No systematic effect of session length on variance estimates was observed (Figure 6, Simulation 5), but increased session lengths do lead to more reliable estimates of $T_{k}$. If variance relates linearly to $T_{k}$, then critical values of $T_{k}$, being a linear function of $S D\left(T_{k}\right)$, relate linearly to the square root of $T_{k}$. Hence, increasing the session length would reduce the proportional difference between the model $T_{k}$ and its upper critical value.

Because the model of Equation 15 uses the coincidence level $k$ as a predictor of $\operatorname{Var}\left(\hat{P}_{k}\right)$, we evaluated possible effects of $k$ on variance estimates. For separate categories of $k$, geometrical averages of $\operatorname{Var}\left(\hat{P}_{k}\right)$ were calculated from Simulations 2-5. Variances were calculated only for $P_{k}$ s below .1. In analogy with Figure 6, ratios of $\operatorname{Var}\left(\hat{P}_{k}\right)_{\text {model }}$ by $\operatorname{Var}\left(P_{k}\right)_{\text {simulation }}$ are presented in Figure 7. Variance ratios are all close to 1 , which suggests an absence of an effect of $k$ on model estimates. No systematic trend of variance ratios over values of $k$ can be observed.
Statistical tests of sniffing task data by QST modeling. The observed panel onset and offset rates (Table 2) produce $\operatorname{Var}\left(\hat{P}_{k}\right)$ values of $.73 \cdot \hat{P}_{k} \cdot k^{-1}$ and $.45 \cdot \hat{P}_{k} \cdot k^{-1}$ for trimmed SP and SF sessions, respectively (Equation 15). The decreased variance estimates for SF sessions must be caused by the increased offset rates, because the increased onset rates could only have increased variance estimates (see Equation 15). In general, changes in offset rates will affect the calculation of variance by Equation 15 more than proportionally identical changes in onset rates. As a consequence of the decreased variance estimates, critical values for $T_{k}$ based on SF sessions are smaller than critical values based on trimmed SP sessions (Table 3). Smaller critical values of $T_{k}$ promote the occurrence of significant effects. Hence, parameter estimates from SF sessions lead to more liberal testing than do estimates from trimmed SP sessions. Due to this, $k=3$ was the lowest coincidence level at which the observed $T$ was significantly above noise $(\alpha=.05$ and $\alpha=.01$ ) for parameter estimates from SF sessions, whereas trimmed SP sessions produced significant $T$ from coincidence level 4 and up (Table 3). Hence, QST noise models indicate that subjects have been responding to at least one stimulus at coincidence level 4 if trimmed SP sessions produced model parameters and at level 3 if SF sessions produced model parameters.

Subsequent post hoc testing of partial $T_{k}$ for separate sniffing peaks showed that the significant results at

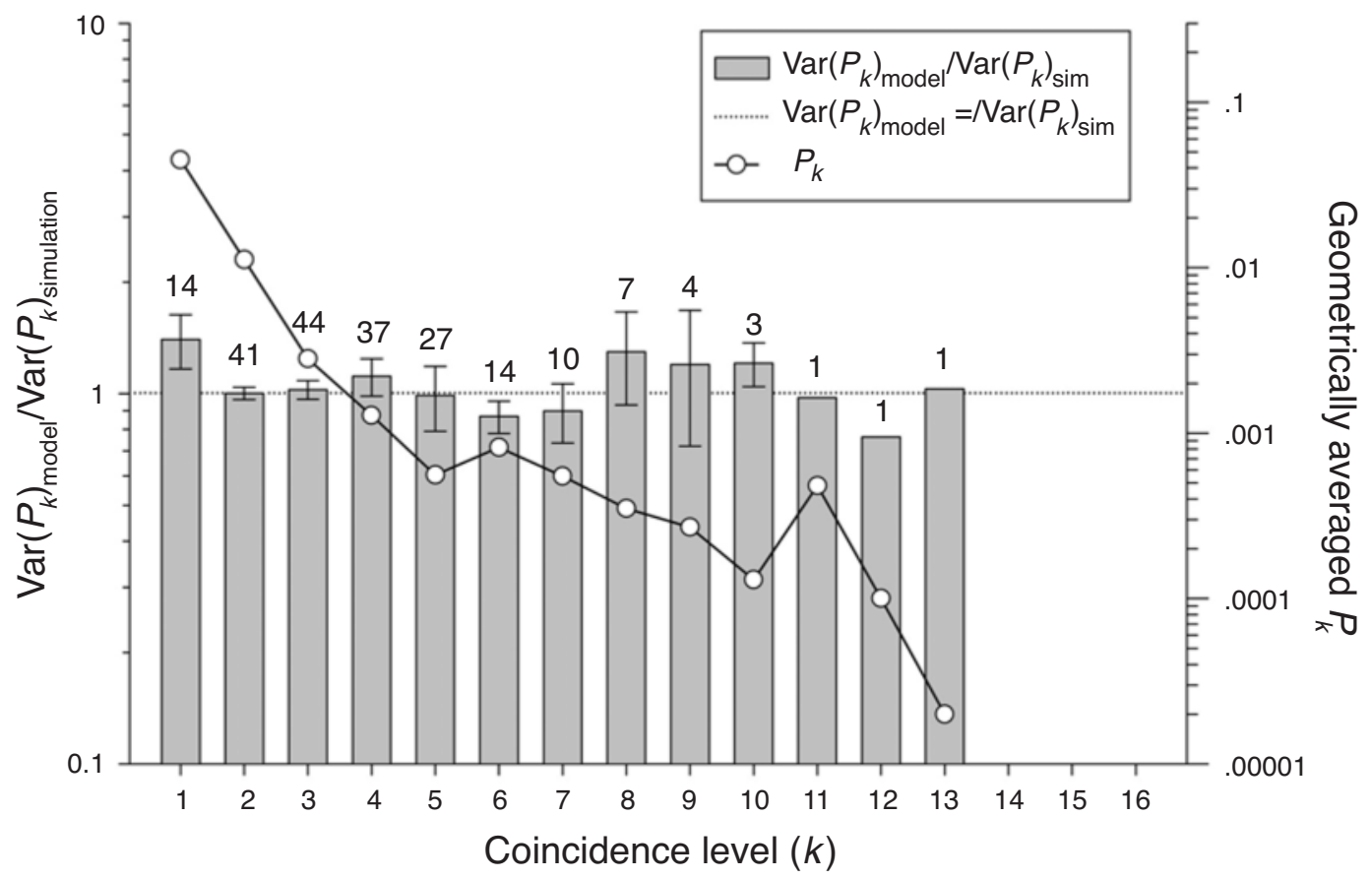

Figure 7. Vertical bars: ratios $( \pm S E M)$ of modeled $\boldsymbol{P}_{\boldsymbol{k}}$ variance (calculated with Equation 15) by simulated $\boldsymbol{P}_{\boldsymbol{k}}$ variance (using the same $\lambda$ and $\mu$ ), plotted as a function of the coincidence level $(k)$. Circles: corresponding geometrically averaged $P_{k}$ at corresponding coincidence levels. Data for which $P_{k}>.1$ are excluded from the analyses. The numbers of observations contributing to the average ratios are presented above the respective bars. 
$k=3$ (SF sessions, $\alpha=.05$ and $\alpha=.01$ ) can be attributed to Stimuli 5, 7, and 8 (Table 5). When the noise model based on trimmed SP sessions was used, Stimuli 5,7 , and 8 were detected at $k=4(\alpha=.05)$ or at $k$ values 5,5 , and $4(\alpha=.01)$, respectively (Table 5$)$. Stimuli 1 and 4 were detected at $k=4$ and 5, respectively, regardless of session context or significance level.

Summarizing, five out of nine stimuli (i.e., 1, 4, 5, 7, and 8 ) were detected unambiguously because they coincided in time with significant sniffing peaks (Figure 3A). Four out of 16 subjects responded simultaneously when Stimulus 3 (isobutyl acetate) was presented. Nonetheless, the short $T_{k, i}$ of this sniffing peak did not result in a significant detection. This illustrates the elementary difference between the QST method and traditional DF methods. The DF methods test whether the observed coincidence level is high enough, whereas the QST method tests whether the duration of coinciding responses lasts long enough at a certain coincidence level. Thereby, it gives secondary importance to the coincidence level.

Of the other three stimuli, two remained undetected, and the status of the third remains ambiguous. The failure to detect Stimulus 2 (propyl acetate) can be attributed to its low concentration, as is suggested by the ratio of mass and detection threshold given in Table 1. Stimulus 9 (hexyl acetate) was not detected by the panel, although its mass/threshold ratio suggests a suprathreshold concentration when compared with other components. The failure of a GCO panel to detect hexyl acetate was observed earlier (Bult et al., 2001). It was attributed to the masking effect of extraneous stimuli that may be gen- erated by the apparatus at high oven temperatures - that is, at relatively high stimulus onset times. These extraneous stimuli can be characterized as burnt smells that may be caused either by column bleeding at high oven temperatures or by the synthesis of volatiles and airborne particles in the casing of the GC oven. Hexyl acetate was released at an oven temperature of approximately $173^{\circ} \mathrm{C}$, a likely oven temperature for extraneous stimuli to be observed. Stimulus 6 (trans-2-hexenal) seems to elicit only two responses. Since this component is released immediately before Stimulus 7, it remains unclear whether some of the responses to Stimulus 7 were, in fact, delayed responses to trans-2-hexenal. An analysis of the qualitative odor descriptions might elucidate this. However, this would go beyond the objectives of the present study.

In the present study, five out of nine stimuli were significantly detected using QST modeling. Using the DF method, Stimulus 4 would have been classified as detected as well, since this stimulus corresponds with a sniffing peak of four coinciding responses (Figure 3B), which exceeds the maximum DF of 3 in the SF session. This does not imply that the QST method lacks sensitivity but, rather, that the traditional DF approach underestimates the probability that short peaks occur accidentally at low coincidences.

\section{GENERAL DISCUSSION}

\section{QST Method}

The primary objective of this study was to develop a method that uses the false alarms generated by a panel in

Table 5

Results of the Post Hoc Analyses of Single Sniffing Peaks, With Significant Peaks Indicated by the Indices of the Coinciding Stimuli (see Figure 3 and Table 1): Lowest Coincidence Level $k$ at Which the Total Response Time $T_{k, i}$ Within That Peak Was Significantly Above Chance Level, Observed $T_{k, i}$, Critical Value $T_{k, i}^{\prime}$ That Constitutes the Minimal Value of Significant $T_{k, i}$, and Onset and Offset Times of the Sniffing Peaks at Corresponding Incidence Levels

\begin{tabular}{|c|c|c|c|c|c|c|c|c|c|c|}
\hline \multirow{2}{*}{$\begin{array}{l}\text { Index of } \\
\text { Coinciding } \\
\text { Stimulus }\end{array}$} & \multicolumn{5}{|c|}{ Stimulus Present } & \multicolumn{5}{|c|}{ Stimulus Free } \\
\hline & $\begin{array}{c}\text { Lowest } \\
\text { Significant } k\end{array}$ & $\begin{array}{c}T_{k, i} \\
\text { Observed (sec) }\end{array}$ & $\begin{array}{c}T_{k, i}^{\prime} \\
(\mathrm{sec})\end{array}$ & $\begin{array}{c}T_{\text {onset }} \\
\text { (min:sec) }\end{array}$ & $\begin{array}{c}T_{\text {offset }} \\
\text { (min:sec) }\end{array}$ & $\begin{array}{c}\text { Lowest } \\
\text { Significant } k\end{array}$ & $\begin{array}{c}T_{k, i} \\
\text { Observed (sec) }\end{array}$ & $\begin{array}{l}T_{k, i}^{\prime} \\
(\mathrm{sec})\end{array}$ & $\begin{array}{c}T_{\text {onset }} \\
\text { (min:sec) }\end{array}$ & $\begin{array}{c}T_{\text {offset }} \\
\text { (min:sec) }\end{array}$ \\
\hline
\end{tabular}

\begin{tabular}{|c|c|c|c|c|c|c|c|c|c|c|}
\hline \multicolumn{11}{|c|}{ Significant Sniffing Peaks at $\alpha=.05$} \\
\hline $1 \mathrm{a}$ & - & - & - & - & - & 4 & 3.3 & 1.7 & $7: 33.4$ & $7: 37.3$ \\
\hline $1 b$ & 4 & 9.0 & 4.2 & $7: 39.4$ & $7: 50.5$ & 4 & 9.0 & 1.7 & $7: 39.4$ & $7: 50.5$ \\
\hline 4 & 5 & 12.2 & 1.2 & $15: 36.4$ & $16: 19.0$ & 5 & 12.2 & 0.4 & $15: 36.4$ & $16: 19.0$ \\
\hline 5 & 4 & 16.6 & 4.2 & $16: 49.4$ & $17: 14.1$ & 3 & 16.6 & 7.0 & $16: 49.3$ & $17: 28.6$ \\
\hline 7 & 4 & 11.8 & 4.2 & $19: 10.7$ & $19: 52.4$ & 3 & 11.8 & 7.0 & $18: 56.3$ & $19: 54.1$ \\
\hline 8 & 4 & 13.8 & 4.2 & $21: 03.0$ & $21: 24.6$ & 3 & 13.8 & 7.0 & $21: 01.4$ & $21: 45.0$ \\
\hline \multicolumn{11}{|c|}{ Significant Sniffing Peaks at $\alpha=.01$} \\
\hline $1 \mathrm{a}$ & - & - & - & - & - & 4 & 3.3 & 3.3 & $7: 33.4$ & $7: 37.3$ \\
\hline $1 b$ & 4 & 9.0 & 7.3 & $7: 39.4$ & $7: 50.5$ & 4 & 9.0 & 3.3 & $7: 39.4$ & $7: 50.5$ \\
\hline 4 & 5 & 12.2 & 2.4 & $15: 36.4$ & $16: 19.0$ & 5 & 12.2 & 0.9 & $15: 36.4$ & $16: 19.0$ \\
\hline 5 & 5 & 4.4 & 2.4 & $16: 49.4$ & $17: 05.7$ & 3 & 16.6 & 11.6 & $16: 49.3$ & $17: 28.6$ \\
\hline 7 & 5 & 11.0 & 2.4 & $19: 10.7$ & $19: 47.9$ & 3 & 11.8 & 11.6 & $18: 56.3$ & $19: 54.1$ \\
\hline 8 & 4 & 9.6 & 7.3 & $21: 03.0$ & $21: 24.6$ & 3 & 13.8 & 11.6 & $21: 01.4$ & $21: 45.0$ \\
\hline
\end{tabular}

Note-Results are presented for tests based on QST parameters obtained from stimulus-present and stimulus-free sessions and for tests at $\alpha=.05$ and $\alpha=.01$. 
a vigilance task to yield noise estimates at a priori significance levels and to include response length as a factor, because stimuli are generally perceived during intervals of time, the length of which is partly related to the probability that a stimulus was actually presented. The QST-based approach meets these objectives and is applicable to conventionally registered timed responses. QST modeling allows for the calculation of critical values $\left(T_{k, i}^{\prime}\right)$ for cumulated response lengths at each coincidence level $k\left(T_{k}\right)$. Upper critical values of $T_{k}^{\prime}$ can be condensed a posteriori into peak-related critical values $T_{k, i}^{\prime}$, allowing the assessment of the minimal coincidence level at which observed $T_{k, i}$ are significant. Since QST does not require structured trials and uses only false alarms, it is easier to apply in vigilance tasks than SDT. Furthermore, the variability of trial length and the use of response length as a factor add to the ecological validity of QST in vigilance task applications. In the present olfactory vigilance experiment, we showed that QST modeling was successful in modeling detection processes.

We think that a noise model based on data from SF sessions is not a good baseline for SP conditions. Critical values of $\hat{T}_{k}$ based on parameters obtained in a SF session were lower than those obtained in a trimmed SP session. Furthermore, post hoc analysis showed that the lowest significant coincidence levels of three out of five sniffing peaks were lower when SF sessions, instead of trimmed SP sessions, were used to estimate the QST noise model (Table 6). However, in the present study these differences would not have led to a different set of significantly detected stimuli. Nevertheless, we propose that noise level estimations for GCO-DF experiments should be based on trimmed SP sessions, rather than on SF sessions.

During SF sessions, the generation of unintended (extraneous) stimuli may cause artificially high coincidence scores. Traditional DF noise estimates based on maximum coincidence levels may be affected easily by such artifacts. In the present vigilance study, coincidence noise levels were calculated from empirical estimates of $\mu$ and $\lambda$ at a priori significance levels. Although estimates of $\mu$ and $\lambda$ from SP sessions may also be affected by panelists' responses to extraneous stimuli, noise estimates will be affected to a much lesser extent, since these are based on global parameters, and not on incidental coincidence scores. As a consequence of the QST model assumption that the occurrences of false alarms of separate observers are not related temporally, actual coincidences are not processed, but only latency and length measures are used. The approximately homogenous distribution of responses over the 30-min sessions shows no evidence of extraneous stimuli in SF or SP sessions (Figure 3). Hence, however small the effect would have been, the QST noise model was not biased by extraneous stimuli in the present study.

In the present study, a maximum of 2 subjects could simultaneously evaluate a particular stimulus sequence. On the other hand, the panel consisted of 16 subjects. To allow for the aggregation of response data of all the subjects, it is required that stimulus sequences and interstimulus intervals be identical for different sessions. Identical stimulus sequences were needed for two reasons. First, responses to identical stimuli in different sessions may fail to coincide if the stimulus onset times differ. Second, stimulus contexts are assumed to be identical for all the subjects. Because slight variations in stimulus onset times did occur, we normalized response times to deal with the problem of noncoinciding responses. One may argue that this does not solve the problem that variations in stimulus onset times may still affect the observed stimulus context. Nonetheless, the average stimulus onset variation of $8.5 \mathrm{sec}$ is considerably smaller than the average interstimulus interval of more than $2 \mathrm{~min}$. Therefore, we argue that stimulus context effects due to stimulus onset variation were negligibly small. Although measures were taken to achieve identical experimental conditions for all the subjects - for example, air conditioning and ventilation - the possible generation of extraneous stimuli by the GC may have constituted a further threat to the uniformity of stimulus sequences. The possible generation of these extraneous stimuli is directly related to the oven temperature of the GC. Because the temperature program was identical for all the subjects, extraneous stimuli, no matter how undesired, could not have created different stimulus contexts between sessions.

\section{Factors Affecting Decision Criteria}

For various modalities, it has been observed that the length of the time period that someone has already been performing a vigilance task correlates negatively with the frequency of both hits and false alarms: Increased watch lengths cause lower response frequencies (Swets, 1977). This effect, which is referred to as the vigilance decrement, is generally attributed to an upward shift in the decision criterion $\beta$, an adaptive response of the subject to a stimulus probability that is lower than anticipated. No such tendency was observed in our SP or SF sessions (Figures $3 \mathrm{~A}$ and $3 \mathrm{~B}$ ). Instead, a rather stable response frequency was observed in both SF windows of SP sessions and in SF sessions. This absence of vigilance decrement may be explained by the fact that the subjects were largely experienced in low stimulus incidence GCO tasks and, hence, were not inclined to adjust their expectations regarding stimulus probability.

The general observation that subjects tend to adjust their response criterion $\beta$ to the observed stimulus probability suggests that observed discrepancies between expected and observed stimulus probabilities are usually attributed externally - that is, to changes in the actual stimulus probabilities. In the present study, the subjects might have attributed discrepancies internally - that is, to weak sensory abilities - if strong expectations of stimulus frequency preexisted. This hypothesis is supported by two other vigilance studies. Williges (1969) made subjects believe that stimulus frequencies were five times higher or lower than the actual stimulus frequency. The 
subjects adapted their $\beta$ to the believed stimulus frequency. Despite the experienced discrepancy between expected and observed stimulus frequencies, the subjects maintained stable $\beta$ throughout the session. In a visual vigilance study, Sullivan and co-workers (Sullivan et al., 1998) reported a drastic increase of false alarm percentage for brain-injured patients in the final phase of the task. In contrast, a control group showed the usual vigilance decrement throughout the task. The authors suggested that the increased false alarm rate of the braininjured patients was due to an internal attribution of the discrepancy between perceived and expected stimulus probability. Hence, the strategy of $\beta$ adjustment may indeed depend on whether stimulus probability discrepancy is attributed internally or externally.

\section{Olfactory Vigilance}

The methodology discussed here relates to continuous vigilance tasks, implying a continuous observation interval. In visual and auditory tasks, this is readily realized by asking a subject to watch a screen constantly or to listen to an audio speaker. Although odors can be presented at any time on a continuous time scale, they cannot be observed continuously. The repetitive breathing pattern of an observer imposes observation intervals on continuous presentations. Inevitably, olfactory presentations are discrete and self-paced by the observer. However, we made an effort to minimize detrimental influences of breathing patterns by giving breathing instructions. The aim of these instructions was to reduce the relative length of the exhalation phase and increase the relative length of the inhalation phase in order to achieve, at best, nearly continuous observations.

A second point that needs to be considered with respect to the use of olfaction in vigilance tasks is that its adaptive function may be fundamentally different from the adaptive functions of vision and audition. Whereas vision and audition permanently guide us in our interactions with our environment, olfaction will often ask for attention only in cases in which immediate action is desirable. The latter involves mental states that tend to be associated with increased vigilance. In animal studies, it was shown that behavioral responses indicating increased vigilance were invoked by unannounced unconditioned presentations of odors signaling either a threat or the presence of food (Dielenberg, Carrive, \& McGregor, 2001; Jones \& Roper, 1997; Terlouw, Boissy, \& Blinet, 1998). In two visual detection studies with human subjects, the presentation of odorants raised vigilance, as was concluded from the increased hit/false alarm ratios (Sullivan et al., 1998; Warm et al., 1991). Results from the studies by Warm et al. and Sullivan et al. suggest that overall vigilance during olfactory tasks may be higher than in auditory or visual tasks. This may explain why no vigilance decrement was observed in this study. Although this failure to find a decrement in vigilance does not affect the data analysis, it may cause differences in typical results of vigilance tasks.
Many vigilance tasks involve the visual or acoustic detection of brief signals. In these tasks, responses merely affirm that stimuli were perceived. These vigilance tasks are in line with the SDT paradigm that assumes short, discrete, and uniform observation intervals and a single yes or no response per interval. In contrast, tasks that involve a continuous evaluation of continuous stimuli are rare in the literature. They occur, however, in many situations in daily life: Do I hear the telephone ringing or not? Do I see a drowning person over there or not? Do I smell leaking gas or not? In the present experiment, hits resulted in sniffing peaks that lasted from approximately $10 \mathrm{sec}$ (Figure 3A, Stimulus 3) up to $1 \mathrm{~min}$ (Figure 3A, Stimuli 4, 5, 7, and 8). To be used in such tasks, SDT would need to account for the information that is enclosed in the length of responses. Therefore, the length of a single hit trial should be variable. Although SDT has been used in continuous vigilance tasks by making post hoc assumptions about the length of observation intervals (Watson $\&$ Nichols, 1976), the extra need for observation intervals to be variable requires fundamental modifications of SDT. For instance, it needs to be resolved how to interpret multiple consecutive hits in response to a single lengthy stimulus. Also, the length of true rejection trials cannot be defined in a straightforward manner, due to the nonuniform length of stimuli. In the present study, we introduced QST as a framework to model noise response behavior. QST merely utilizes false alarms and no hits, and it includes response length as a contributor to stimulus detection probability. Therefore, in continuous vigilance tasks with variable response length, QST is a good alternative to the fundamentally less appropriate SDT.

\section{REFERENCES}

Acree, T. E., Barnard, J., \& Cunningham, D. G. (1984). A procedure for the sensory analysis of gas chromatographic effluents. Food Chemistry, 14, 273-286.

BORLAND SOFTWARE CORPORATION (1998). Delphi, Version 4.1. Scotts Valley, CA: Author.

Bult, J. H. F., Schifferstein, H. N. J., Roozen, J. P., VoraGeN, A. G. J., \& Kroeze, J. H. A. (2001). The influence of olfactory concept on the probability of detecting sub- and peri-threshold components in a mixture of odorants. Chemical Senses, 26, 459-469.

BUtTery, R. G., \& Ling, L. C. (1998). Additional studies on flavor components of tortilla chips. Journal of Agricultural \& Food Chemistry, 46, 2764-2769.

Colquhoun, W. P., \& Baddeley, A. D. (1964). Role of pretest expectancy in vigilance decrement. Journal of Experimental Psychology, 68, 156-160.

Colqu Houn, W. P., \& BAdDeley, A. D. (1967). Influence of signal probability during pretraining on vigilance decrement. Journal of Experimental Psychology, 73, 153-155.

CraIg, A. (1987). Signal detection theory and probability matching apply to vigilance. Human Factors, 29, 645-652.

Da Silva, M. A. A. P., Lundahl, D. S., \& McDaniel, M. R. (1994). The capability and psychophysics of Osme: A new GC-olfactometry technique. In H. Maarse \& D. G. Van der Heij (Eds.), Trends in flavour research: Proceedings of the 7th Weurman Flavour Research Symposium, Noordwijkerhout, The Netherlands (pp. 15-32). Amsterdam: Elsevier.

Dielenberg, R. A., Carrive, P., \& McGregor, I. S. (2001). The car- 
diovascular and behavioral response to cat odor in rats: Unconditioned and conditioned effects. Brain Research, 897, 228-237.

Dravnieks, A., \& O'Donnell, A. (1971). Principles and some techniques of high-resolution headspace analysis. Journal of Agricultural \& Food Chemistry, 19, 1049-1056.

EgAN, J. P., GreenberG, G. Z., \& SCHulman, A. I. (1961a). Interval of time uncertainty in auditory detection. Journal of the Acoustical Society of America, 33, 771-778.

Egan, J. P., GreenberG, G. Z., \& Schulman, A. I. (1961b). Operating characteristics, signal detectability, and the method of free response. Journal of the Acoustical Society of America, 33, 993-1007.

Flath, R. A., Black, D. R., Guadagni, D. G., McFadden, W. H., \& ScHultz, T. H. (1967). Identification and organoleptic evaluation of compounds in Delicious apple essence. Journal of Agricultural \& Food Chemistry, 15, 29-35.

GREEN, D. M., \& SwETS, J. A. (1966). Signal detection theory and psychophysics (1st ed.). New York: Wiley.

Grosch, W. (2001). Evaluation of the key odorants of foods by dilution experiments, aroma models and omission. Chemical Senses, 26, 533545.

Hall, G., \& Andersson, J. (1983). Volatile fat oxidation products: I. Determination of odour thresholds and odour intensity functions by dynamic olfactometry. Lebensmittel Wissenschaft und Technologie, 16, 354-361.

Jones, R. B., \& Roper, T. J. (1997). Olfaction in the domestic fowl: A critical review. Physiology \& Behavior, 62, 1009-1018.

Kleinrock, L. (1975). Queueing systems (1st ed.). New York: Wiley.

Kollmannsberger, H., \& Berger, R. G. (1992). Precursor atmosphere storage induced flavour changes in apples cv. red delicious. Chemie Mikrobiologie Technologie der Lebensmittel, 14, 81-86.

Le Guen, S., Prost, C., \& Demaimay, M. (2000). Critical comparison of three olfactometric methods for the identification of the most potent odorants in cooked mussels (Mytilus edulis). Journal of Agricultural \& Food Chemistry, 48, 1307-1314.

LuCE, R. D. (1986). Response times: Their role in inferring elementary mental organization (1st ed.). Oxford: Oxford University Press.

Maarse, H., Visscher, C. A., Willemsens, L. C., \& Boelens, M. H. (1989). Volatile compounds in food (6th ed.). Zeist, The Netherlands: TNO-CIVO.

MACKWORTH, J. F., \& TAYLOR, M. M. (1963). The $d^{\prime}$ measure of signal detectability in vigilance-like situations. Canadian Journal of Psychology, 17, 302-325.

MCDaniel, M. R., Miranda-Lopez, R., Watson, B. T., Micheals, N. J., \& LibBey, L. M. (1990). Pinot noir aroma: A sensory/gas chromatographic approach. In G. Charalambous (Ed.), Flavors and off-flavors: Proceedings of the 6th International Flavor Conference, Rhethymnon, Crete, Greece (pp. 23-26). Amsterdam: Elsevier.

ONG, P. K. C., \& ACREE, T. E. (1998). Gas chromatography/olfactory analysis of lychee (Litchi chinesis Sonn.). Journal of Agricultural \& Food Chemistry, 46, 2282-2286.

OtT, A., FAy, L. B., \& Chaintreau, A. (1997). Determination and origin of the aroma impact compounds of yogurt flavor. Journal of Agricultural \& Food Chemistry, 45, 850-858.

Pollien, P., Ott, A., Montigon, F., Baumgartner, M., MuñozBox, R., \& Chaintreau, A. (1997). Hyphenated headspace-gas chromatography-sniffing technique: Screening of impact odorants and quantitative aromagram. Journal of Agricultural \& Food Chemistry, 45, 2630-2637.

Press, W. H., Flannery, B. P., Teukolsky, S. A., \& Vetterling, W. T. (1989). Numerical recipes in Pascal (1st ed.). New York: Cambridge University Press.

Rychlik, M., Schieberle, P., \& Grosch, W. (1998). Compilation of odor thresholds, odor qualities and retention indices of key food odorants (1st ed.). Garching, Germany: Deutsche Forschungsanstalt für Lebensmittelchemie and Institut für Lebensmittelchemie der Technischen Universität München.

See, J. E., Warm, J. S., Dember, W. N., \& Howe, S. R. (1997). Vigilance and signal detection theory: An empirical evaluation of five measures of response bias. Human Factors, 39, 14-29.

Sullivan, T. E., Warm, J. S., Schefft, B. K., Dember, W. N., O'Dell, M. W., \& Peterson, S. J. (1998). Effects of olfactory stimulation on the vigilance performance of individuals with brain injury. Journal of Clinical \& Experimental Neuropsychology, 20, 227-236.

SwETs, J. A. (1961). Detection theory and psychophysics: A review. Psychometrika, 26, 49-63.

SwETS, J. A. (1977). Signal detection theory applied to vigilance. In R. R. Mackie (Ed.), Vigilance: Theory, operational performance and physiological correlates (pp. 705-718). New York: Plenum.

TAKeOKa, G. R., Buttery, R. G., Ling, L. C., Wong, R. Y., DAO, L. T., EDWARDS, R. H., \& BERRIOS, J. D. J. (1998). Odor thresholds of various unsaturated branched esters. Lebensmittel Wissenschaft und Technologie, 31, 443-448.

Takeoka, G. R., Buttery, R. G., Turnbaugh, J. G., \& Teranishi, R. (1991). Cyclic esters: Compounds possessing remarkably low odor thresholds. Lebensmittel Wissenschaft und Technologie, 24, 569-570.

Terlouw, E. M. C., Boissy, A., \& Blinet, P. (1998). Behavioural responses of cattle to the odours of blood and urine from conspecifics and to the odour of faeces from carnivores. Applied Animal Behaviour Science, 57, 9-21.

ULLRICH, F., \& GROSCH, W. (1987). Identification of most intense volatile flavor compounds formed during autoauxidation of linoleic acid. Zeitschrift für Lebensmittel Untersuchung und Forschung, 184, $277-282$.

van Gemert, L. J., \& Nettenbreijer, A. H. (1977). Compilation of odour threshold values in air and water. Voorburg, The Netherlands: National Institute for Water Supply RID and Central Institute for $\mathrm{Nu}-$ trition and Food Research TNO.

VAN RUTH, S. M., \& Roozen, J. P. (1994). Gas chromatography/sniffing port analysis and sensory evaluation of commercially dried bell peppers (Capsicum annuum) after rehydration. Food Chemistry, 51, 165170.

van Ruth, S. M., Roozen, J. P., \& CoziJnsen, J. L. (1995). Volatile compounds of rehydrated French beans, bell peppers and leeks: I. Flavour release in the mouth and in three mouth model systems. Food Chemistry, 53, 15-22.

vaN Ruth, S. M., ROOZEN, J. P., \& CoZIJnsen, J. L. (1996). Gas chromatograpy/sniffing port analysis evaluated for aroma release from rehydrated French beans (Phaseolus vulgaris). Food Chemistry, 56, 343-346.

ViCKers, D., Leary, J., \& Barnes, P. (1977). Adaptation to decreasing signal probability. In R. R. Mackie (Ed.), Vigilance: Theory, operational performance and physiological correlates (pp. 679-703). New York: Plenum.

Warm, J. S., Dember, W. N., \& Parasuraman, R. (1991). Effects of olfactory stimulation on performance and stress in a visual sustained attention task. Journal of the Society of Cosmetic Chemists, 42, 199210.

Watson, C. S., \& Nichols, T. L. (1976). Detectability of auditory signals presented without defined observation intervals. Journal of the Acoustical Society of America, 59, 655-668.

WILLIGES, R. C. (1969). Within-session criterion changes compared to an ideal observer criterion in a visual monitoring task. Journal of Experimental Psychology, 81, 61-66.

Winer, B. J., Brown, D. R., \& Michels, K. M. (1991). Statistical principles in experimental design (pp. 354-358). New York: McGrawHill. 


\section{APPENDIX \\ Consequences of Assuming a Gaussian, Rather Than the More Realistic Gamma, $P_{K}$ Distribution}

We investigated the impact of assuming $P_{k}$ to be Gaussian distributed on the size of critical values of $P_{k}$ by substituting a skewed gamma distribution for the Gaussian distribution, keeping means and variances unchanged. Gamma distributions are typically unimodal and positively skewed. The standard gamma distribution function defines probability density $P(t)$ by

$$
P(t)=\frac{\beta^{\alpha} t^{\alpha-1} e^{-\beta t}}{\Gamma(\alpha)} \quad(\alpha, \beta>0),
$$

where $t$ equals time ( $\min )$ and $\Gamma(\alpha)$ is defined by

$$
\Gamma(\alpha)=\int_{0}^{\infty} t^{\alpha-1} e^{-t} d t \quad(t \in \mathbb{R}),
$$

When $\alpha$ equals 1, Equation A1 simplifies to the one-parameter exponential function of Equation 1, describing a very skewed distribution. For higher values of ?, the density distribution becomes increasingly more symmetrical, approximating the Gaussian distribution. Mean $(M)$ and variance (Var) of the gamma distribution are given by $\alpha / \beta$ and $\alpha / \beta^{2}$, respectively. We use the sample standard deviation $(S D)$ divided by the sample mean as an index of skewness: higher $S D / M$ ratios (close to 1 or higher) imply that $S D$ is larger than the mean, which limits the $P_{k}$ distribution unilaterally for low $P_{k}$. Alternatively, low $S D / M$ ratios $(0.1$ or less) imply symmetrical $P_{k}$ distributions. This is illustrated in Figure A1 for three different $S D / M$ ratios that were found for a 20-subject panel and 1,000 replicated simulations with $\lambda=0.25$ and $\mu=10$.

From the gamma distribution's mean and variance definitions, $\alpha$ and $\beta$ can be derived so that the mean and the standard deviation of the gamma distribution equal those of the Gaussian:

$$
\alpha=\left(\frac{M}{S D}\right)^{2},
$$

showing unequivocally the relation between $\alpha$ and skewness, and

$$
\beta=\frac{M}{S D^{2}} \text {. }
$$

Distributions of $\hat{T}_{k}$ can be modeled as $P_{k}$ distributions by using Equation 7 . In the left column of Figure A1, three examples of $\hat{T}_{k}$ distributions show the skewness of three examples of means and standard deviations of $\hat{T}_{k}$ that were observed in the simulations. It is shown that the discrepancy between the shapes of the Gaussian and the gamma distributions increases for increasing skewness indices - that is, at decreasing mean $\hat{T}_{k}$ values. Consequently, critical values of $T_{k}$ depend on the assumed distribution of $\hat{T}_{k}$ (Figure A1, right column). We calculated critical values of Gaussian-distributed $T_{k}$ at one-sided 5\% and $1 \%$ significance levels for skewness indexes ranging from 0.01 to 12.8 . For these critical $T_{k}$ values, corresponding true cumulative probabilities of the gamma distribution were calculated. These true probabilities of gamma-distributed $T_{k}$ values are shown for various skewness indices in Figure A2.

Figure A2 shows that the difference between Gaussian- and gamma-based confidence levels at certain $T_{k} \mathrm{~s}$ is highest for gamma distributions that approximate the exponential function ( $\alpha$ values close to 1$)$. At skewness index 1.12, $\alpha$ equals 0.81 (Equation A4, Figure A1), resulting in a very skewed distribution with a concurring average $T_{k}$ of $0.069 \mathrm{~min}(4.15 \mathrm{sec})$, assuming a session time of $60 \mathrm{~min}$. This implies that large skewness occurs at $T_{k}$ levels that will be very distinguishing with respect to whether a response coincidence should be considered a signal or not. At $T_{k}$ s below $0.069 \mathrm{~min}$ - that is, at skewness indices larger than 1.12 - critical values for gamma-distributed $T_{k}$ increase to values close to, or even larger than, Gaussian-distributed $T_{k}$. For these $T_{k} \mathrm{~s}$, assuming a Gaussian distribution of $T_{k}$ does not lead to less conservative testing.

We conclude that critical values of $T_{k}$ should be based on gamma distributions, rather than on Gaussian distributions. If one decides to work with Gaussian distributions, then Figure A2 may give an indication of the adjusted confidence levels one should take into account. Ignoring this, however, will introduce a bias toward more liberal testing at the most relevant coincidence intervals. 


\section{APPENDIX (Continued)}

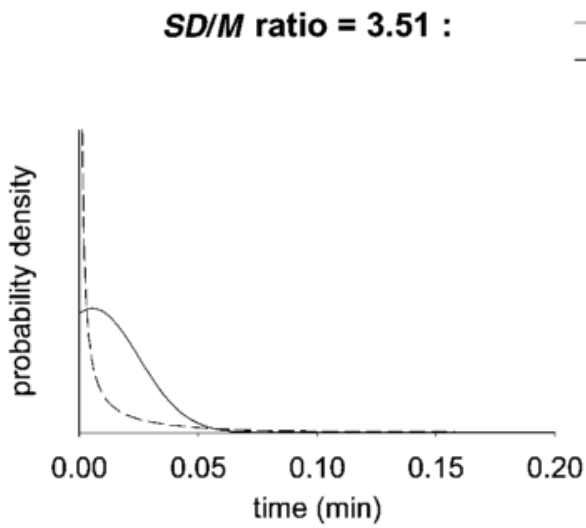

Gaussian: Mean $=0.0055(\mathrm{~min}), S D=0.019(\mathrm{~min})$

Gamma: $\alpha=0.081, \beta=14.63$

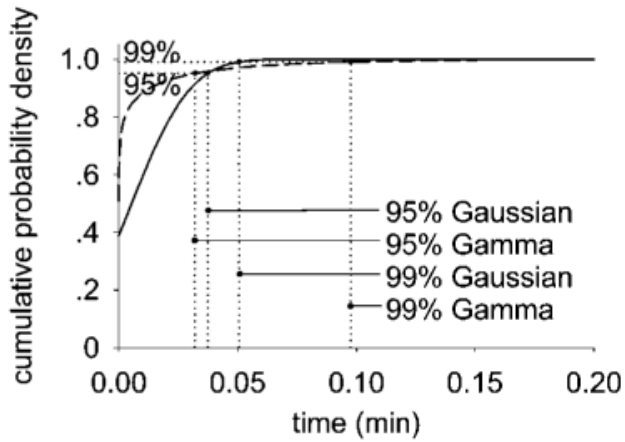

$S D / M$ ratio $=0.42:$

Gaussian: Mean $=0.65(\mathrm{~min}), S D=0.27(\mathrm{~min})$

Gamma: $\alpha=5.73, \beta=8.78$
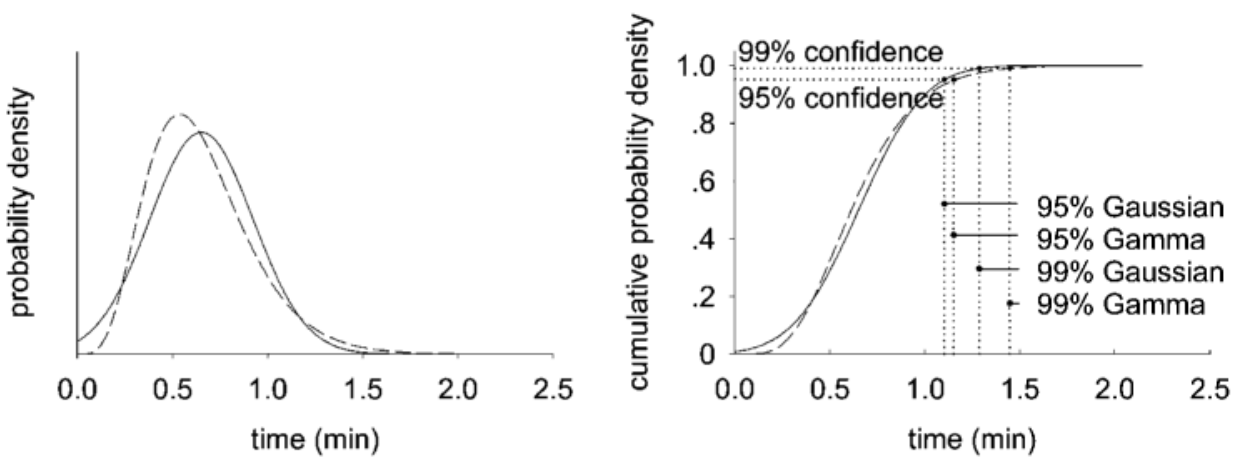

$S D / M$ ratio $=0.014:$

Gaussian: Mean $=18.3(\mathrm{~min}), S D=2.50(\mathrm{~min})$

Gamma: $\alpha=53.6, \beta=2.93$
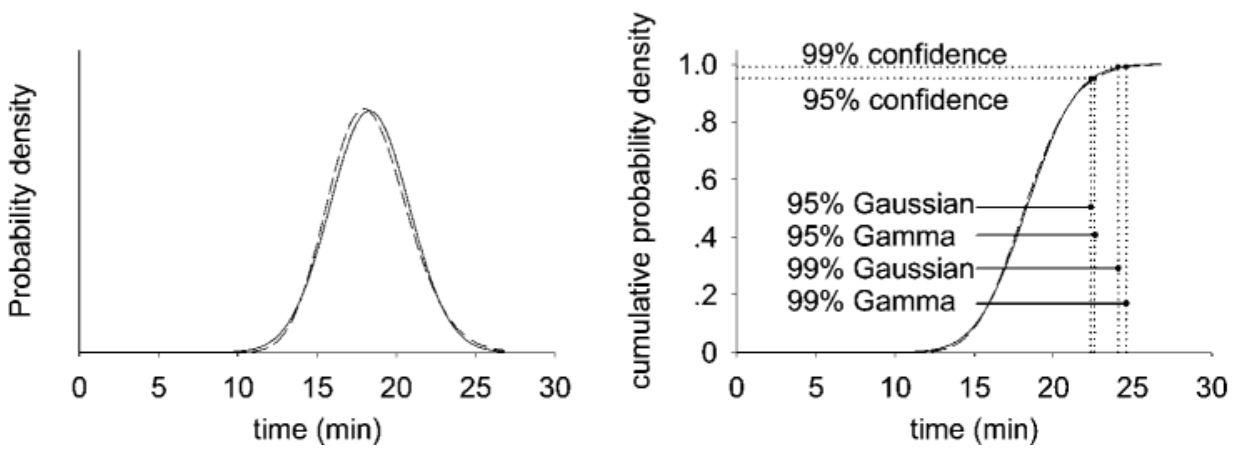

Figure A1. Gaussian and gamma density distributions with equal means and variances and corresponding cumulative density distributions for three skew levels. The standard deviation by mean ratios $(S D / M) 3.51,0.42$, and 0.014 indicate high, medium, and low skew, respectively. 
APPENDIX (Continued)

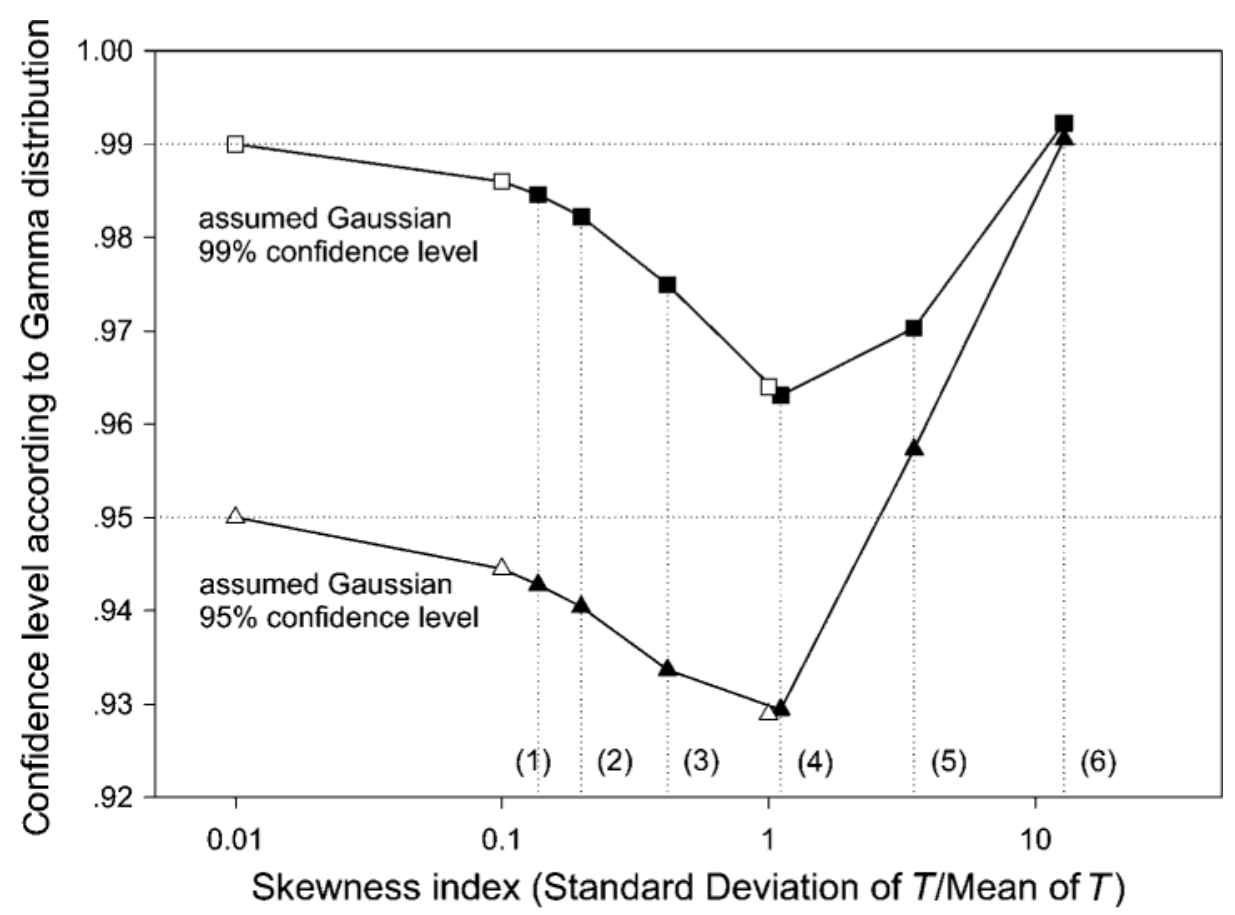
(1) - Mean $T=18.31 \mathrm{~min}$
(2) - Mean $T=4.35 \mathrm{~min}$
(3) - Mean $T=0.65 \mathrm{~min}$
(4) - Mean $T=0.069 \mathrm{~min}$
(5) - Mean $T=0.006 \mathrm{~min}$
$\neg-95 \%$ assumed, added for illustration purpose
- $95 \%$ assumed, simulation data
$-\square-99 \%$ assumed, added for illustration purpose
$\rightarrow-99 \%$ assumed, simulation data

Figure A2. True confidence level of $T_{k}$ (using the gamma distribution) corresponding to the $99 \%$ and $95 \%$ confidence levels incorrectly calculated using a Gaussian distribution, plotted as a function of the skewness index. Gamma and Gaussian distributions have identical means and variances.

(Manuscript received August 15, 2002;

revision accepted for publication January 5, 2004.) 